\title{
A SPARSE GRAHAM-ROTHSCHILD THEOREM
}

\author{
HANS JÜRGEN PRÖMEL AND BERND VOIGT
}

\begin{abstract}
The main result of this paper is a sparse version of the GrahamRothschild partition theorem for $n$-parameter sets [R. L. Graham and B. L. Rothschild, Ramsey's theorem for n-parameter sets, Trans. Amer. Math. Soc. 159 (1971), 257-292]. In particular, a sparse version of Hales-Jewett's theorem is proved. We give several applications, e.g., for arithmetic progressions and finite sums of integers, confirming conjectures of J. Spencer and of J. Nešetril and V. Rödl.

We also consider graphs defined on parameter sets and prove a sparse and restricted induced partition theorem for such graphs, extending results from [H. J. Prömel, Induced partition properties of combinatorial cubes, J. Combin. Theory Ser. A 39 (1985), 177-208] and [P. Frankl, R. L. Graham, and V. Rödl, Induced restricted Ramsey theorems for spaces, J. Combin. Theory Ser. A 44 (1987), 120-128].
\end{abstract}

1. Introduction. In their fundamental paper [1971] R. L. Graham and B. L. Rothschild introduced the concept of parameter sets and established a Ramsey theorem for parameter sets. Compare, e.g., Prömel, Voigt [1986] for an account on recent developments.

The idea to study graphs which are defined on parameter sets is quite natural and apparently has been considered first in Deuber, Rothschild and Voigt [1982] generalizing an analogous approach of J. Spencer [1975] for arithmetic progressions. A Ramsey type partition theorem for parameter sets (an induced Graham-Rothschild theorem) has been established, then, in Prömel [1985].

Another line of research considers so-called 'restricted' questions. E.g., to get a monochromatic $m$-parameter subset no complete $(m+1)$-parameter set should be necessary. With respect to the Hales-Jewett theorem, which is the case of partitioning 0-parameter sets, a restricted theorem has been proved in Deuber, Prömel, Rothschild and Voigt [1984].

Recently, P. Frankl, R. L. Graham and V. Rödl [1987] proved an induced and restricted version of the Graham-Rothschild theorem. The Frankl-Graham-Rödl paper pioneers applying the Nešetril-Rödl amalgamation technique to the structure of parameter sets. The arguments are considerably short and clear, covering particular cases of Prömel's induced Graham-Rothschild theorem and even establishing a restricted result thereof. Still another line of research considers so-called 'sparse' versions. E.g., to obtain a monochromatic $m$-parameter subset no short cycles of $m$-parameter sets should be necessary.

Received by the editors June 1,1987 .

1980 Mathematics Subject Classification (1985 Revision). Primary 05A99; Secondary 05C55, $10 \mathrm{~A} 50$. 
Using the probabilistic method, V. Rödl [1981] established a sparse version of the Hales-Jewett theorem.

In this paper we combine all three approaches and prove a sparse and restricted induced partition theorem for graphs on parameter sets. Our main technique is that of amalgamations. We call it the Nešetrill-Rödl-technique, as similar ideas have been used extensively by J. Nešetřil and V. Rödl to prove Ramsey type results for ordinary graphs which are defined on sets, compare, e.g., Prömel and Voigt [1987] for an account.

The paper is organized as follows: In $\S 2$ we explain the concept of parameter sets, resp., parameter words. In $\S 3$ we state our main theorem, a Sparse GrahamRothschild Theorem. $\S \S 4-8$ contain a proof thereof. In $\S 4$ an auxiliary graphtheoretic result is explained. $\S 5$ introduces the basic notion for our proof, that of partite configurations. In $\S 6$ we explain how to amalgamate partite configurations. Relevant properties of the amalgamation are given in $\S 7$. Finally, the proof itself is given in $\S 8$. Although it contains some delicate details, the basic plan follows ideas from Nešetrril and Rödl [1984]. $\S 9$ contains applications and corollaries of the Sparse Graham-Rothschild Theorem. In particular, the existence of sparse van der Waerden and sparse Rado sets is shown. This section can be read independently from $\S \S 4-8$. In terms of graphs which are defined on parameter sets the Sparse Graham-Rothschild Theorem still restricts to considering complete graphs, lacking a general result which covers, e.g., also Prömel's induced Graham-Rothschild theorem. In $\S \S 10-14$ we approach such a sparse and induced Graham-Rothschild theorem. On the way we achieve a simultaneously restricted result. In $\S 10$ the notion of graphs on parameter sets is described. In $\S 11$ we carry over the tools from $\S \S 5$ and 6 , introducing partite graphs and their amalgamation. $\S 12$ shows how the ideas from $\S 8$ can be adapted to prove the induced Graham-Rothschild theorem. $\S 13$ gives the crucial definition of connectedness that is needed later on, in $\S 14$, to formulate the Sparse and Restricted Induced Graham-Rothschild Theorem. This, then, is the final result of our paper. Its proof follows the patterns of $\S \S 8$ and 12 .

2. Parameter sets and parameter words. Let $A$ be a fixed finite and nonempty set. We are interested in $A^{n}$, the set of $n$-tuples over $A$, and certain subsets (parameter sets). 0-parameter sets are simply singleton elements of $A^{n}$. In general, an m-parameter set $M \subseteq A^{n}$ is given by an m-parameter word $f=$ $\left(f_{0}, \ldots, f_{n-1}\right) \in\left(A \cup\left\{\lambda_{0}, \ldots, \lambda_{m-1}\right\}\right)^{n}$. We require that each parameter $\lambda_{i}, i<$ $m$, occurs at least once in $f$. In order to avoid ambiguities we assume that $A \cap$ $\left\{\lambda_{i} \mid i<m\right\}=\varnothing$; the set of elements $a \in A$ should be distinguished from the set of parameters $\lambda_{i}, i<m$. For $f \in\left(A \cup\left\{\lambda_{0}, \ldots, \lambda_{m-1}\right\}\right)^{n}$ and $g \in\left(A \cup\left\{\lambda_{0}, \ldots, \lambda_{k-1}\right\}\right)^{m}$ the composition $f \cdot g \in\left(A \cup\left\{\lambda_{0}, \ldots, \lambda_{k-1}\right\}\right)^{n}$ is the word resulting from replacing the parameter $\lambda_{i}$ in $f$ by $g_{i}$, the $i$ th component of $g$. Note that this composition is well defined, regardless of $f$, resp. $g$, being parameter words. If, however, $f$ and $g$ are parameter words then so is $f \cdot g$. In particular, for $k=0$, we obtain a set $M=\left\{\left(f \cdot\left(a_{0}, \ldots, a_{m-1}\right) \mid\left(a_{0}, \ldots, a_{m-1}\right) \in A^{m}\right\} \subseteq A^{n}\right.$. This is the $m$-parameter set related to $f$. Clearly, two parameter words yield the same parameter set if they differ only by a permutation of their parameters. We get a rigid representation requiring the first occurrences of different parameters to be in increasing order, first $\lambda_{0}$, then $\lambda_{1}$, etc. We give a formal definition. 
Let us denote by $[A]\left(\begin{array}{l}n \\ m\end{array}\right)$ the set of all words (mappings) $f: n \rightarrow A \cup\left\{\lambda_{0}, \ldots\right.$, $\left.\lambda_{m-1}\right\}$ satisfying

for every $j<m$ there exists $i<n$ with $f(i)=\lambda_{j}$, and $\min f^{-1}\left(\lambda_{i}\right)<\min f^{-1}\left(\lambda_{j}\right)$ for all $i<j<m$.

Mappings $f \in[A]\left(\begin{array}{l}n \\ m\end{array}\right)$ are called m-parameter words of length $n$ over $A$. For $f \in$ $[A]\left(\begin{array}{l}n \\ m\end{array}\right)$ and $g \in[A]\left(\begin{array}{c}m \\ k\end{array}\right)$ the composition $f \cdot g \in[A]\left(\begin{array}{l}n \\ k\end{array}\right)$ is defined as above, i.e., $(f \cdot g)(i)=f(i)$ if $f(i) \in A$ and $(f \cdot g)(i)=g(j)$ if $f(i)=\lambda_{j}$.

Observe that $[A]\left(\begin{array}{l}n \\ 0\end{array}\right)=A^{n}$. For $f \in[A]\left(\begin{array}{l}n \\ m\end{array}\right)$ the set $M=\left\{f \cdot g \mid g \in[A]\left(\begin{array}{c}m \\ 0\end{array}\right)\right\}=$ $f \cdot[A]\left(\begin{array}{c}m \\ 0\end{array}\right)$ is the $m$-parameter subset of $A^{n}$ described by $f$. Note that we have defined parameter words also with respect to one-element sets $A$, although the interpretation of parameter sets as subsets of $A^{n}$ does not make sense. However, parameter words $f \in[\{0\}]\left(\begin{array}{l}n \\ k\end{array}\right)$ represent families of $k$ nonempty and disjoint subsets of $\{0, \ldots, n-1\}$, viz., $f^{-1}\left(\lambda_{i}\right), i<k$. Then $f \cdot[\{0\}]\left(\begin{array}{l}k \\ 1\end{array}\right)$ is the set of all unions of these $k$ sets. Using the language of extremal problems, $[\{0\}]\left(\begin{array}{l}n \\ k\end{array}\right)$ is the set of strong $\Delta$-systems with $k$ terms.

The Graham-Rothschild partition theorem for parameter sets (resp., parameter words) asserts that

For every triple $k, m$ and $r$ of nonnegative integers there exists a positive integer $n$ such that

$$
\left\{\begin{array}{l}
\text { for every coloring } \Delta:[A]\left(\begin{array}{l}
n \\
k
\end{array}\right) \rightarrow\{0, \ldots, r-1\} \text { there exists } \\
\text { an } f \in[A]\left(\begin{array}{c}
n \\
m
\end{array}\right) \text { such that } \Delta(f \cdot g)=\Delta(f \cdot h) \text { for all } \\
g, h \in[A]\left(\begin{array}{c}
m \\
k
\end{array}\right) .
\end{array}\right.
$$

We abbreviate $(*)$ by $n \stackrel{[A]}{\longrightarrow}(m)_{r}^{k}$.

REMARK. With respect to $k=0$ this is the Hales-Jewett theorem (Hales and Jewett [1963]). For $k>0$ the original proof appeared in Graham and Rothschild [1971], for shorter ones see Deuber and Voigt [1982] or Spencer [1979].

By abuse of language we do not distinguish between parameter sets and the corresponding parameter words and simply refer to $[A]\left(\begin{array}{l}n \\ m\end{array}\right)$ as the set of m-spaces in $A^{n}$. For an $m$-space $f \in[A]\left(\begin{array}{l}n \\ m\end{array}\right)$ the set $f \cdot[A]\left(\begin{array}{c}m \\ k\end{array}\right)$, then, is the set of $k$-spaces in $f$. So the assertion (*) says that for every $r$-coloring of the $k$-spaces in $A^{n}$ there exists an $m$-space with all its $k$-spaces in the same color (provided that $n \geq n(r, k, m)$ is large enough).

3. A Sparse Graham-Rothschild Theorem. The notions of an $m$-space induced by a set of $i$-spaces and of cycles of $m$-spaces with respect to $k$-spaces are crucial for our results. Let $\mathscr{F} \subseteq[A]\left(\begin{array}{c}n \\ i\end{array}\right)$ be a set of $i$-spaces in $A^{n}$. An $m$-space $f \in[A]\left(\begin{array}{l}n \\ m\end{array}\right)$ is induced by $\mathscr{F}$ if $f \cdot[A]\left(\begin{array}{c}m \\ i\end{array}\right) \subseteq \mathscr{F}$ (all its $i$-spaces belong to $\mathscr{F}$ ). By $\{\mathscr{F}\}_{m}$ we denote the set of all $m$-spaces which are induced by $\mathscr{F}$, in other words,

$$
\{\mathscr{F}\}_{m}=\left\{f \in[A]\left(\begin{array}{c}
n \\
m
\end{array}\right) \mid f \cdot[A]\left(\begin{array}{c}
m \\
i
\end{array}\right) \subseteq \mathscr{F}\right\} .
$$

Next we introduce the concept of cycles with respect to intersections in $k$-spaces. Let $\mathscr{G} \subseteq[A]\left(\begin{array}{l}n \\ m\end{array}\right)$ be a set of $m$-spaces in $A^{n}$ and let $k \leq m$. A $k$-cycle of length $c$ in $\mathscr{G}$ is given by a sequence $f_{0}, \ldots, f_{c-1}$ of mutually distinct $m$-spaces $f_{\nu} \in \mathscr{G}$ and a sequence $h_{0}, \ldots, h_{c-1}$ of mutually distinct $k$-spaces $h_{\nu} \in[A]\left(\begin{array}{l}n \\ k\end{array}\right)$ such that $h_{\nu} \in f_{\nu} \cdot[A]\left(\begin{array}{c}m \\ k\end{array}\right) \cap f_{\nu+1} \cdot[A]\left(\begin{array}{c}m \\ k\end{array}\right)$ for $\nu<c-1$ and $h_{c-1} \in f_{c-1} \cdot[A]\left(\begin{array}{c}m \\ k\end{array}\right) \cap f_{0} \cdot[A]\left(\begin{array}{c}m \\ k\end{array}\right)$. 
In particular, a $k$-cycle of length two is given by two distinct $m$-spaces $f_{0}, f_{1} \in \mathscr{G}$ sharing two distinct $k$-spaces, in particular, $f_{0}$ and $f_{1}$ share a $(k+1)$-space.

By $k$-girth $\mathscr{G}$ we denote the length of the shortest $k$-cycle in $\mathscr{G}$.

The main result of this paper is the

Sparse Graham-RothSChild TheOREM. (a) Let $A$ be a one-element set. Let $k, m, r$ and $c$ be positive integers. Then there exists a positive integer $n$ and there exists a set $\mathscr{F} \subseteq[A]\left(\begin{array}{l}n \\ 1\end{array}\right)$ of 1-spaces such that

(1) $k$-girth $\{\mathscr{F}\}_{m}>c$, and

(2) $\left(n,\{\mathscr{F}\}_{m}\right) \rightarrow(m)_{r}^{k}$, meaning that for every $r$-coloring $\Delta:\{\mathscr{F}\}_{k} \rightarrow\{0, \ldots$, $r-1\}$ there exists an $f \in\{\mathscr{F}\}_{m}$ such that $\Delta(f \cdot g)=\Delta(f \cdot h)$ for all $g, h \in[A]\left(\begin{array}{c}m \\ k\end{array}\right)$.

(b) Let $A$ be a finite set with $|A| \geq 2$. Let $m, r$ and $c$ be positive integers and let $k<m$ be a nonnegative integer such that $m \cdot|A| \geq 3$. Then there exists a positive integer $n$ and there exists a set $\mathscr{F} \subseteq A^{n}$ such that

(1) $k$-girth $\{\mathscr{F}\}_{m}>c$, and

(2) $\left(n,\{\mathscr{F}\}_{m}\right) \rightarrow(m)_{r}^{k}$, with the same meaning as before.

REMARKS. (1) The condition $m \cdot|A| \geq 3$ just excludes the case $k=0, m=1$ and $|A|=2$ for which the result does not hold. Here only the weaker result mentioned below is valid, compare also the result of $\S 14$.

(2) Using the probabilistic method a weaker result can be established, however, its proof is somewhat simpler (Rödl, [1981]; Rucinski, Voigt [xx]): given $k, m, r$ and $c$ as above there exists a family $\mathscr{E} \subseteq[A]\left(\begin{array}{l}n \\ m\end{array}\right)$ of $m$-spaces such that for every $r$-coloring of the $k$-spaces in $A^{n}$ there exists a monochromatic $f \in \mathscr{E}$ but still $\mathscr{E}$ does not contain short $k$-cycles. Unfortunately, such families $\mathscr{E}$ obtained by the probabilistic method cover far more $k$-spaces than desired, viz., these $k$-spaces, then, induce additional $m$-spaces, creating even cycles of length two.

(3) The essential feature of our result is that the $m$-spaces are induced by spaces of lowest possible dimension. It is somewhat astonishing that with just one exception this is always possible. A weaker result would be, e.g., to require only a family $\mathscr{F} \subseteq[A]\left(\begin{array}{l}n \\ k\end{array}\right)$ of $k$-spaces satisfying (1) and (2).

4. A sparse product lemma. As a tool for proving the Sparse GrahamRothschild Theorem we need a certain sparse and induced version of the product pigeon hole principle. This result has been proved in Prömel and Voigt [1987].

Notation. For sets $P_{s}$ and $Q_{s}, s<t$, we denote by $\left\{P_{s} \rightarrow Q_{s} \mid s<t\right\}$ the set of mappings $\pi: \bigcup_{s<t} P_{s} \rightarrow \bigcup_{s<t} Q_{s}$ such that $\pi(\beta) \in Q_{s}$ for $\alpha \in P_{s}$ (the sets $P_{s}$ should be mutually disjoint).

Notation. Let $p$ be a positive integer, let $m_{0}, \ldots, m_{p-1}$ be positive integers and let $P_{s}$ and $Q_{s}$ for $s<t$ be nonempty sets. A $p$-partite graph on $\prod_{\mu<p} m_{\mu}$ with values in $\left\{P_{s} \rightarrow Q_{s} \mid s<t\right\}$ is given by a mapping $\mathscr{G}: \prod_{\mu<p} m_{\mu} \rightarrow\left\{P_{s} \rightarrow Q_{s} \mid s<t\right\}$.

Let $\tilde{\mathscr{G}}: \prod_{\mu<p} \tilde{m}_{\mu} \rightarrow\left\{P_{s} \rightarrow Q_{s} \mid s<t\right\}$ be another $p$-partite graph with values in $\left\{P_{s} \rightarrow Q_{s} \mid s<t\right\}$. Let $X_{\mu} \in\left[\tilde{m}_{\mu}\right]^{m_{\mu}}$ and let $f_{\mu}: m_{\mu} \rightarrow \tilde{m}_{\mu}$ be the monotone injection such that $X_{\mu}=\left\{f_{\mu}(k) \mid k<m_{\mu}\right\}$. We say that $\prod_{\mu<p} X_{\mu}$

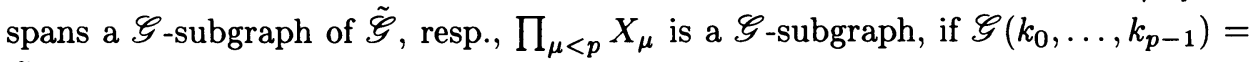
$\tilde{\mathscr{G}}\left(f_{0}\left(k_{0}\right), \ldots, f_{p-1}\left(k_{p-1}\right)\right)$ for all $\left(k_{0}, \ldots, k_{p-1}\right) \in \prod_{\mu<p} m_{\mu}$.

Note that for every $g \in \bigcup_{s<t} P_{s}$ there exists a subset $I \subseteq\{0, \ldots, p-1\}$, possibly empty, such that $\mathscr{G}\left(k_{0}, \ldots, k_{p-1}\right)(g)=\mathscr{G}\left(k_{0}^{\prime}, \ldots, k_{p-1}^{\prime}\right)(g)$ whenever $k_{\mu}=k_{\mu}^{\prime}$ for 
all $\mu \in I$. As the set of such $I$ is closed under intersections there exists a minimal such $I$ which is denoted by $I(g)$, resp. $\tilde{I}(g)$, if we consider $\tilde{G} . I(g)$ is the set of coordinates on which the $\mathscr{G}$-value of $g$ actually depends.

SPARSE PRODUCT LemMa. Let $\mathscr{G}: \prod_{\mu<p} m_{\mu} \rightarrow\left\{P_{s} \rightarrow Q_{s} \mid s<t\right\}$ be a $p$ partite graph with values in $\left\{P_{s} \rightarrow Q_{s} \mid s<t\right\}$, let $\pi$ be an element of $\left\{P_{s} \rightarrow\right.$ $\left.Q_{s} \mid s<t\right\}$ and let $r$ and $c$ be positive integers. Then there exists a p-partite graph $\tilde{\mathscr{G}}: \prod_{\mu<p} \tilde{m}_{\mu} \rightarrow\left\{P_{s} \rightarrow Q_{s} \mid s<t\right\}$ and there exists a family $\mathscr{S}$ of $\mathscr{G}$-subgraphs of $\tilde{\mathscr{G}}$ such that

(1) $I(g)=\tilde{I}(g)$ for all $g \in \bigcup_{s<t} P_{s}$,

(2) girth $\mathscr{S}>c$, where the girth is defined in the usual way, considering $\mathscr{S}$ as a family of subsets of $\prod_{\mu<p} \tilde{m}_{\mu}$,

(3) for every coloring $\Delta: \tilde{\mathscr{G}}^{-1}(\pi) \rightarrow\{0, \ldots, r-1\}$ there exists a $\mathscr{G}_{\text {-subgraph }}$ $\prod_{\mu<p} X_{\mu} \in \mathscr{S}$ such that $\Delta\left(k_{0}, \ldots, k_{p-1}\right)=\Delta\left(k_{0}^{\prime}, \ldots, k_{p-1}^{\prime}\right)$ for all $\left(k_{0}, \ldots, k_{p-1}\right)$ and $\left(k_{0}^{\prime}, \ldots, k_{p-1}^{\prime}\right)$ in $\prod_{\mu<p} X_{\mu}$ with $\tilde{\mathscr{G}}\left(k_{0}, \ldots, k_{p-1}\right)=\tilde{\mathscr{G}}\left(k_{0}^{\prime}, \ldots, k_{p-1}^{\prime}\right)=\pi$, i.e., $\Delta\rceil\left(\tilde{\mathscr{G}}^{-1}(\pi) \cap \prod_{\mu<p} X_{\mu}\right)$ is a constant coloring.

Notation. Assertion (3) is abbreviated as $(\tilde{\mathscr{G}}, \mathscr{S}) \rightarrow(\mathscr{G})_{r}^{\pi}$.

5. Partite configurations. The concatenation of finite sequences is denoted by $\otimes$, i.e., $\left(x_{0}, \ldots, x_{m-1}\right) \otimes\left(y_{0}, \ldots, y_{n-1}\right)=\left(x_{0}, \ldots, x_{m-1}, y_{0}, \ldots, y_{n-1}\right)$. Let us agree that $i$ denotes a nonnegative integer which is fixed throughout this and the next section. Let $A_{+}=A \cup\left\{\lambda_{0}, \ldots, \lambda_{i-1}\right\}$. Typically, $i=0$ or $i=1$, although for later applications also other $i$ are of interest. For $|A|=1$ we shall tacitly assume that $i \geq 1$.

An m-partite $i$-configuration in $A^{n}$ is a family $\left(\mathscr{M}_{g}\right)_{g \in[A]\left(\begin{array}{c}m \\ i\end{array}\right)}$ of subsets $\mathscr{M}_{g} \subseteq$ $A_{+}^{n}$. Shorthand we write $\mathscr{M}_{g \in[A]\left(\begin{array}{c}m \\ i\end{array}\right)} \subseteq A_{+}^{n}$. An embedding between partite $i$ configurations $\mathscr{M}_{g \in[A]\left(\begin{array}{c}m \\ i\end{array}\right)} \subseteq A_{+}^{n}$ and $\tilde{\mathscr{M}}_{g \in[A]\left(\begin{array}{c}\tilde{m} \\ i\end{array}\right)} \subseteq A_{+}^{\tilde{n}}$ is given by an $f \in[A]\left(\begin{array}{c}\tilde{m}+\tilde{n} \\ m+n\end{array}\right)$ satisfying $f \cdot\left(g \otimes \mathscr{M}_{g}\right) \subseteq g^{*} \otimes \tilde{\mathscr{M}}_{g^{*}}$ for all $g \in[A]\left(\begin{array}{c}m \\ i\end{array}\right)$, where $\left.g^{*}=(f\rceil \tilde{m}\right) \cdot g$. In particular, we require that $f\rceil \tilde{m} \in[A]\left(\begin{array}{c}\tilde{m} \\ m\end{array}\right)$, where $\left.f\right\rceil \tilde{m}$ is the initial segment of $f$ consisting of the first $\tilde{m}$ entries. If $f$ is an embedding we write $f: \mathscr{M}_{g \in[A]\left(\begin{array}{c}m \\ i\end{array}\right)} \hookrightarrow$ $\tilde{\mathscr{M}}_{g \in[A]\left(\begin{array}{c}\tilde{m} \\ i\end{array}\right)}$. Observe that an embedding $f \in[A]\left(\begin{array}{c}\tilde{m}+\tilde{n} \\ m+n\end{array}\right)$ can be decomposed as $f=$ $(f\rceil \tilde{m}) \otimes f_{\text {tail }}$, where $f_{\text {tail }} \in\left(A \cup\left\{\lambda_{i} \mid i<m+n\right\}\right)^{\tilde{n}}$ is such that the parameters $\lambda_{m}, \ldots, \lambda_{m+n-1}$ occur in $f_{\text {tail }}$ and their first occurrences are in increasing order. Using this decomposition we see that $f_{\text {tail }} \cdot\left(g \otimes \mathscr{M}_{g}\right) \subseteq \tilde{\mathscr{M}}_{g^{*}}$. Hence, the family $\left(f_{\text {tail }} \cdot\left(g \otimes \mathscr{M}_{g}\right)\right)_{g \in[A]\left(\begin{array}{c}m \\ i\end{array}\right)}$ is an $m$-partite $i$-subconfiguration of $\tilde{\mathscr{M}}_{g \in[A]\left(\begin{array}{c}\tilde{m} \\ i\end{array}\right)}$ which can be viewed as the image of the embedding $f$. Every $h \in[A]\left(\begin{array}{c}\tilde{m} \\ k\end{array}\right)$ gives rise to a $k$-partite $i$-configuration $\mathscr{M}_{g \in[A]\left(\begin{array}{c}k \\ i\end{array}\right)} \subseteq A_{+}^{\tilde{n}}$, namely the preimage of the embedding

$$
h \otimes\left(\lambda_{k}, \ldots, \lambda_{k+\tilde{n}-1}\right): \mathscr{M}_{g \in[A]\left(\begin{array}{l}
k \\
i
\end{array}\right)} \hookrightarrow \tilde{\mathscr{M}}_{g \in[A]\left(\begin{array}{c}
\tilde{m} \\
i
\end{array}\right)} .
$$

More precisely, $\mathscr{M}_{g}=\tilde{\mathscr{M}}_{h \cdot g}$. By abuse of language we denote this $k$-partite $i$ configuration by $\tilde{\mathscr{M}}_{h \cdot g \in h \cdot[A]\left(\begin{array}{l}k \\ i\end{array}\right)}$. 
More generally, if $f \in[A]\left(\begin{array}{c}\tilde{m}+\tilde{n} \\ m+n\end{array}\right)$ is such that $\left.f\right\rceil m \in[A]\left(\begin{array}{c}\tilde{m} \\ m\end{array}\right)$ then $f$ induces the $m$-partite $i$-configuration $\mathscr{\mathscr { M }}_{g \in[A]\left(\begin{array}{c}m \\ i\end{array}\right)} \subseteq A_{+}^{n}$ which is defined by

$$
\mathscr{M}_{g}=\left\{h \in A_{+}^{n} \mid f \cdot(g \otimes h) \in \mathscr{M}_{(f\rceil \tilde{m}) \cdot g}\right\} .
$$

We say that an $l$-space $f \in[A]\left(\begin{array}{c}\tilde{m}+\tilde{n} \\ l\end{array}\right)$ is induced by the $\tilde{m}$-partite $i$-configuration $\tilde{\mathscr{M}}_{g \in[A]\left(\begin{array}{c}\tilde{m} \\ i\end{array}\right)}$ if $f \cdot[A]\left(\begin{array}{l}l \\ i\end{array}\right)$ is a subset of $\bigcup_{g \in[A]\left(\begin{array}{c}\tilde{m} \\ i\end{array}\right)} g \otimes \tilde{\mathscr{M}}_{g}$. By abuse of language we denote by $\left\{\tilde{\mathscr{M}}_{g \in[A]\left(\begin{array}{c}\tilde{m} \\ i\end{array}\right)}\right\}_{l}$ the set of $l$-spaces which are induced by $\tilde{\mathscr{M}}_{g \in[A]}\left(\begin{array}{c}\tilde{m} \\ i\end{array}\right)$. Note that for $i \geq 1$ this necessarily implies that $f\rceil \tilde{m} \in[A]\left(\begin{array}{c}\tilde{m} \\ l\end{array}\right)$. In general we say that $f$ is crossing if $f\rceil \tilde{m} \in[A]\left(\begin{array}{c}\tilde{m} \\ l\end{array}\right)$.

6. Amalgamations of partite configurations. In this section we describe the concept of amalgamation. Let $\mathscr{M}_{g \in[A]\left(\begin{array}{c}m \\ i\end{array}\right)} \subseteq A_{+}^{n}$ be an $m$-partite $i$-configuration and let $k$ be an integer such that $i \leq k \leq m$. Let $h \in[A]\left(\begin{array}{c}m \\ k\end{array}\right)$ be a $k$-space in $A^{m}$. Recall that $\mathscr{M}_{h \cdot g \in h \cdot[A]\left(\begin{array}{l}k \\ i\end{array}\right)} \subseteq A_{+}^{n}$ is a $k$-partite $i$-configuration. Let $\tilde{\mathscr{N}}_{g \in[A]\left(\begin{array}{l}k \\ i\end{array}\right)} \subseteq$ $A_{+}^{\tilde{n}}$ be another $k$-partite $i$-configuration and let $\mathscr{E}=\left\{e_{0}, \ldots, e_{z-1}\right\}$ be a set of embeddings

$$
e_{j}: \mathscr{M}_{h \cdot g \in h \cdot[A]\left(\begin{array}{l}
k \\
i
\end{array}\right)} \hookrightarrow \tilde{\mathscr{N}}_{g \in[A]\left(\begin{array}{l}
k \\
i
\end{array}\right)} .
$$

We want to define the amalgamation of $\mathscr{M}_{g \in[A]\left(\begin{array}{c}m \\ i\end{array}\right)}$ with $\left(\tilde{\mathscr{N}}_{g \in[A]\left(\begin{array}{c}k \\ i\end{array}\right)}, \mathscr{E}\right)$ along $h$, which results in an $m$-partite $i$-configuration

$$
\mathscr{N}_{g \in[A]\left(\begin{array}{c}
m \\
i
\end{array}\right)}=\mathscr{M}_{g \in[A]\left(\begin{array}{c}
m \\
i
\end{array}\right)} *_{h}\left(\tilde{\mathscr{N}}_{g \in[A]\left(\begin{array}{c}
k \\
i
\end{array}\right)}, \mathscr{E}\right) .
$$

See the figure.

We need two auxiliary lemmas.

LEMMA 1. Let $h \in[A]\left(\begin{array}{c}m \\ k\end{array}\right)$ be a $k$-space in $A^{m}$ and let $z$ be a positive integer. Then there exist parameter words

$$
f_{j} \in[A]\left(\begin{array}{c}
(1+z) \cdot m \\
m
\end{array}\right)
$$

for $j<z$ with the following properties:

(1) $f_{j} \cdot x=f_{j^{\prime}} \cdot x$ for all $j<j^{\prime}<z$ and all $x \in h \cdot A^{k}$, but $f_{j} \cdot x \neq f_{j^{\prime}} \cdot x^{\prime}$ for all $j<j^{\prime}<z$ and all $x \in A^{m} \backslash h \cdot A^{k}$ and all $x^{\prime} \in A^{m}$;

(2) $f_{j} \cdot g=f_{j^{\prime}} \cdot g$ for all $j<j^{\prime}<z$ and all $g \in h \cdot[A]\left(\begin{array}{l}k \\ 1\end{array}\right)$, but $f_{j} \cdot g \neq f_{j^{\prime}} \cdot g^{\prime}$ for all $j<j^{\prime}<z$ and all $g \in[A]\left(\begin{array}{c}m \\ 1\end{array}\right) \backslash h \cdot[A]\left(\begin{array}{c}k \\ 1\end{array}\right)$ and all $g^{\prime} \in[A]\left(\begin{array}{c}m \\ 1\end{array}\right)$;

(3) for every

$$
g \in[A](\underset{2}{(1+z) \cdot m})
$$

such that $g \cdot[A]\left(\begin{array}{l}2 \\ 1\end{array}\right) \subseteq \bigcup_{j<z} f_{j} \cdot[A]\left(\begin{array}{c}m \\ 1\end{array}\right)$ but $g \cdot[A]\left(\begin{array}{l}2 \\ 1\end{array}\right) \nsubseteq \bigcup_{j<z} f_{j} \cdot h \cdot[A]\left(\begin{array}{l}k \\ 1\end{array}\right)$ there exists $j<z$ such that $g \cdot[A]\left(\begin{array}{c}2 \\ 1\end{array}\right) \subseteq f_{j} \cdot[A]\left(\begin{array}{c}m \\ 1\end{array}\right)$;

(4) assume that $|A| \geq 3$, then for every

$$
g \in[A]\left(\begin{array}{c}
(1+z) \cdot m \\
1
\end{array}\right)
$$

such that $g \cdot A \subseteq \bigcup_{j<z} f_{j} \cdot A^{m}$ but $g \cdot A \nsubseteq \bigcup_{j<z} f_{j} \cdot h \cdot A^{k}$ there exists $j<z$ such that $g \cdot A \subseteq f_{j} \cdot A^{m}$. 
(a)

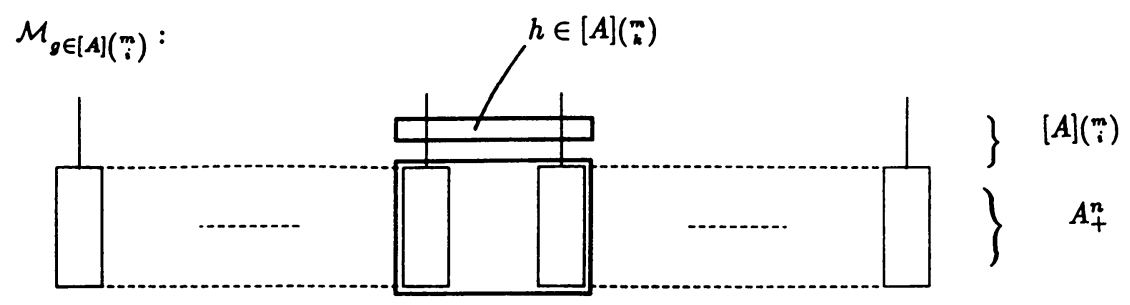

(b)

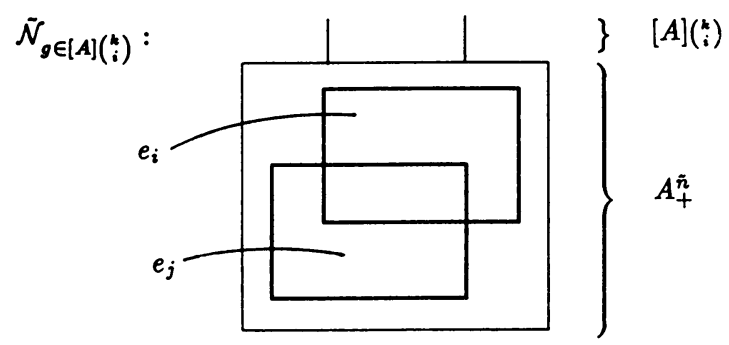

(c) $\mathcal{N}_{g \in[A]\left(\begin{array}{l}n \\ i\end{array}\right)}:$

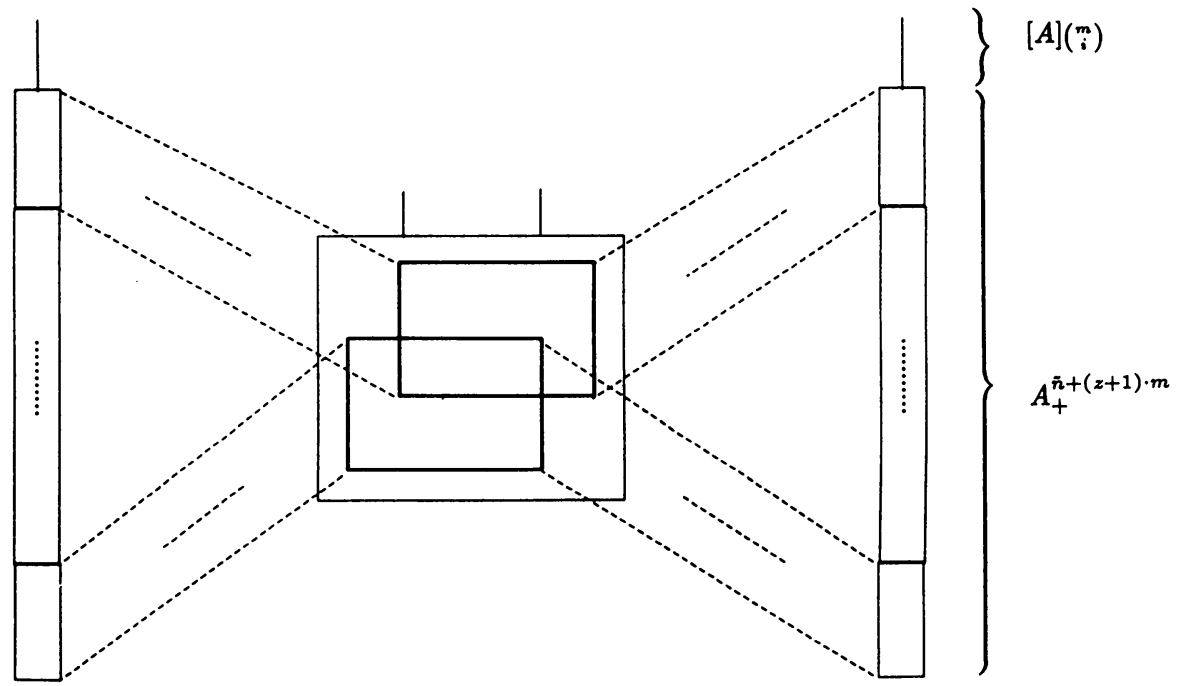

REMARK. For sets $A$ with at least two elements assertion (2) follows readily from (1). For sets with at least three elements assertion (3) follows from (4). In human language, (1), resp. (2), assert that the $m$-spaces $f_{j}$ mutually intersect in the $k$-space $h$ and have nothing else in common. Moreover, (3), resp. (4), show that any 2-space, resp. one-space, if $|A| \geq 3$, which is contained in the union of the $f_{j}$ but which is not a subspace of $h$ already belongs to one of the $m$-spaces $f_{j}$.

PROOF OF LEMMA 1. Say, $h=\left(h_{0}, \ldots, h_{m-1}\right)$. For $j<k$ let the number $j_{0}$ be defined by $j_{0}=\min \left\{i<m \mid h_{i}=\lambda_{j}\right\}$. As $h \in[A]\left(\begin{array}{c}m \\ k\end{array}\right)$ the $j_{0}$ are well defined. 
Consider $\tilde{h}=\left(\tilde{h}_{0}, \ldots, \tilde{h}_{m-1}\right) \in\left(A \cup\left\{\lambda_{j_{0}} \mid j<k\right\}\right)^{m}$ which is defined by

$$
\begin{aligned}
\tilde{h}_{i} & =h_{i} & & \text { if } h_{i} \in A, \\
& =\lambda_{j_{0}} & & \text { if } h_{i}=\lambda_{j} .
\end{aligned}
$$

Let $\vec{\lambda}_{m}=\left(\lambda_{0}, \ldots, \lambda_{m-1}\right)$. Now we define $f_{j} \in[A]\left(\begin{array}{c}\left(\begin{array}{c}1+z \\ m\end{array}\right) \cdot m \\ m\end{array}\right)$ by

$$
f_{j}=\vec{\lambda}_{m} \otimes \underbrace{\tilde{h} \otimes \cdots \otimes \tilde{h}}_{j \text {-times }} \otimes \vec{\lambda}_{m} \otimes \underbrace{\tilde{h} \otimes \cdots \otimes \tilde{h}}_{(z-1-j) \text {-times }} .
$$

Observe that for every $g \in[A]\left(\begin{array}{c}m \\ i\end{array}\right)$ we have that $h \cdot g=g$ if and only if $g \in h \cdot[A]\left(\begin{array}{c}k \\ i\end{array}\right)$. This readily implies assertions (1) and (2).

We now prove (3). Let

$$
g \in[A]\left(\begin{array}{c}
(1+z) \cdot m \\
2
\end{array}\right)
$$

be as in (3). First observe that already $\bar{g}=g\rceil m \in[A]\left(\begin{array}{c}m \\ 2\end{array}\right)$ and, moreover, $\bar{g} \notin$ $h \cdot[A]\left(\begin{array}{c}k \\ 2\end{array}\right)$. Hence, $\tilde{h} \cdot \bar{g} \neq \bar{g}$. Let $a$ be an element of $A$. As the two 1-subspaces $g \cdot\left(a, \lambda_{0}\right)$ and $g \cdot\left(\lambda_{0}, a\right)$ uniquely determine $g$, in particular, $\bar{g} \times\left(a, \lambda_{0}\right)$ and $\bar{g} \times\left(\lambda_{0}, a\right)$ determine $\bar{g}$, it suffices to show that for some $j<z$ both $g \cdot\left(a, \lambda_{0}\right)$ and $g \cdot\left(\lambda_{0}, a\right)$ are elements of $f_{j} \cdot[A]\left(\begin{array}{c}m \\ 1\end{array}\right)$. So we have to show that for some $j<z$

$$
\begin{gathered}
g \cdot\left(\hat{a}, \lambda_{0}\right)=\bar{g} \cdot\left(a, \lambda_{0}\right) \otimes \underbrace{\tilde{h} \cdot \bar{g} \cdot\left(a, \lambda_{0}\right) \otimes \cdots \otimes \tilde{h} \cdot \bar{g} \cdot\left(a, \lambda_{0}\right)}_{j} \otimes \bar{g} \cdot\left(a, \lambda_{0}\right) \\
\otimes \underbrace{\tilde{h} \cdot \bar{g} \cdot\left(a, \lambda_{0}\right) \otimes \cdots \otimes \tilde{h} \cdot g \cdot\left(a, \lambda_{0}\right)}_{z-1-j}
\end{gathered}
$$

and

$$
\begin{aligned}
g \cdot\left(\lambda_{0}, a\right)= & \bar{g}\left(\lambda_{0}, a\right) \otimes \underbrace{\tilde{h} \cdot g \cdot\left(\lambda_{0}, a\right) \otimes \cdots \otimes \tilde{h} \cdot \bar{g}\left(\lambda_{0}, a\right)}_{j} \otimes \bar{g}\left(\lambda_{0}, a\right) \\
& \otimes \underbrace{\tilde{h} \cdot \bar{g} \cdot\left(\lambda_{0}, a\right) \otimes \cdots \otimes \tilde{h} \cdot \bar{g} \cdot\left(\lambda_{0}, a\right)}_{z-1-j}
\end{aligned}
$$

belong to $f_{j} \cdot[A]\left(\begin{array}{c}m \\ 1\end{array}\right)$. This follows as $\tilde{h} \cdot \bar{g} \neq \bar{g}$.

Assertion (4) can be verified using an analogous reasoning. Observe that we have to use essentially the fact that $|A| \geq 3$, i.e., every 1 -space contains at least three 0-subspaces.

The significance of the next lemma will be clear in the construction of the amalgamation. The problem is the following: consider the embedding $e_{j}: \mathscr{M}_{h \cdot g \in h \cdot[A]\left(\begin{array}{l}k \\ i\end{array}\right)} \hookrightarrow$ $\tilde{\mathscr{W}}_{g \in[A]\left(\begin{array}{c}k \\ k\end{array}\right)}$, so $e_{j} \in[A]\left(\begin{array}{c}k+\tilde{n} \\ k+n\end{array}\right)$. We want to find an $e_{j}^{*} \in[A]\left(\begin{array}{c}m+\tilde{n} \\ m+n\end{array}\right)$ such that $e_{j}^{*}$. $\left(h \otimes\left(\lambda_{k}, \ldots, \lambda_{k+n-1}\right)\right)$ behaves like $e_{j}$.

LEMMA 2. Let $e \in[A]\left(\begin{array}{c}k+\tilde{n} \\ k+n\end{array}\right)$ be such that e can be written as $e=\left(\lambda_{0}, \ldots, \lambda_{k-1}\right)$ $\otimes e_{\text {tail }}$. Let $h \in[A]\left(\begin{array}{c}m \\ k\end{array}\right)$. Then there exists $e^{*} \in[A]\left(\begin{array}{c}m+\tilde{n} \\ m+n\end{array}\right)$ which can be written as $e^{*}=\left(\lambda_{0}, \ldots, \lambda_{m-1}\right) \otimes e_{\text {tail }}^{*}$ such that for all $f \in[A]\left(\begin{array}{c}k+n \\ i\end{array}\right)$ it follows that

$$
\left.e^{*} \cdot\left(\left(h \otimes\left(\lambda_{k}, \ldots, \lambda_{k+n-1}\right)\right) \cdot f\right)=(h \cdot f\rceil k\right) \otimes\left(e_{\text {tail }} \cdot f\right) .
$$


PROOF OF LEMMA 2. The construction of $e^{*}$ is straightforward. Again, for $j<k$ let $j_{0}=\min \left\{\nu<m \mid h_{\nu}=\lambda_{j}\right\}$. Say, $e_{\text {tail }}=\left(\alpha_{0}, \ldots, \alpha_{\tilde{n}-1}\right)$. Then define $e^{*}=\left(\lambda_{0}, \ldots, \lambda_{m-1}\right) \otimes\left(\alpha_{0}^{*}, \ldots, \alpha_{\tilde{n}-1}^{*}\right)$, where

$$
\begin{aligned}
\alpha_{\nu}^{*} & =\alpha_{\nu} & & \text { if } \alpha_{\nu} \in A, \\
& =\lambda_{m+j} & & \text { if } \alpha_{\nu}=\lambda_{k+j}, \\
& =\lambda_{j_{0}} & & \text { if } \alpha_{\nu}=\lambda_{j} \text { for } j<k .
\end{aligned}
$$

This obviously has the desired properties.

Now we can define the $*_{h}$-amalgamation. For this purpose let the parameter words

$$
f_{j} \in[A]\left(\begin{array}{c}
(z+1) \cdot m \\
m
\end{array}\right)
$$

for $j<z$ be as in Lemma 1. Also let $e_{j}^{*} \in[A]\left(\begin{array}{c}m+\tilde{n} \\ m+n\end{array}\right)$ be as in Lemma 2 with respect to $h$ and $e_{j}$. For $g \in[A]\left(\begin{array}{c}m \\ i\end{array}\right)$ define $\mathscr{N}_{g} \subseteq A_{+}^{\tilde{n}+(z+1) \cdot m}$ by

$$
g \otimes \mathscr{N}_{g}=\bigcup_{j<z} e_{j}^{*} \cdot\left(g \otimes \mathscr{M}_{g}\right) \otimes f_{j} \cdot g .
$$

In particular, let $\mathscr{M}_{g}^{j}$ be defined by $g \otimes \mathscr{M}_{g}^{j}=e_{j}^{*} \cdot\left(g \otimes \mathscr{M}_{g}\right) \otimes f_{j} \cdot g$. Note that

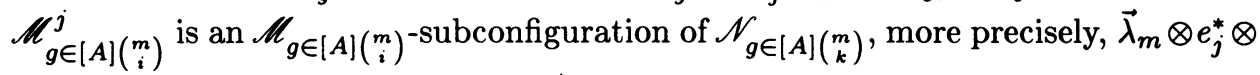
$f_{j}: \mathscr{M}_{g \in[A]\left(\begin{array}{c}m \\ i\end{array}\right)} \hookrightarrow \mathscr{N}_{g \in[A]\left(\begin{array}{c}m \\ i\end{array}\right)}$ has $\mathscr{M}_{g \in[A]\left(\begin{array}{c}m \\ i\end{array}\right)}^{j}$ as its image; compare figure (c). Note also that $\mathscr{N}_{h \cdot g \in h \cdot[A]\left(\begin{array}{l}k \\ i\end{array}\right)}$ is isomorphic to $\tilde{\mathscr{N}}_{g \in[A]\left(\begin{array}{l}k \\ i\end{array}\right)}$, i.e., recall that for each $g \in[A]\left(\begin{array}{l}k \\ i\end{array}\right)$ it follows that

$$
\mathscr{N}_{h \cdot g}=\tilde{\mathscr{N}_{g}} \otimes \underbrace{g \otimes \cdots \otimes g}_{(z+1) \text { times }} .
$$

REMARK. Observe that the spaces $e_{j}^{*}$ and $f_{j}$ which are used in the amalgamation do not depend on $i$.

7. Some properties of amalgamations. In this section we give some properties of the $*_{h}$-amalgamation. We use the same notation as in the preceding section.

AMALGAMATION PROPERTY. For $g \in[A]\left(\begin{array}{c}m \\ i\end{array}\right) \backslash h \cdot[A]\left(\begin{array}{c}k \\ i\end{array}\right)$ we have for all $j^{\prime}<j<z$ that $\mathscr{M}_{g}^{j^{\prime}} \cap \mathscr{M}_{g}^{j}=\varnothing$.

PROOF. Obvious, cf. Lemma 1 together with our tacit assumption that $i \geq 1$ for $|A|=1$.

This property actually justifies calling $*_{h}$ an amalgamation, it also shows that figure (c) gives an appropriate depiction of $\mathscr{N}_{g \in[A]}\left(\begin{array}{c}m \\ i\end{array}\right)$.

Recall that $\left\{\tilde{\mathscr{N}}_{g \in[A]\left(\begin{array}{l}k \\ i\end{array}\right)}\right\}_{k}$ is the set of induced $k$-spaces.

COLORING PROPERTY. Assume that $\left(\tilde{\mathscr{N}}_{g \in[A]\left(\begin{array}{c}k \\ i\end{array}\right)}, \mathscr{E}\right) \rightarrow\left(\mathscr{M}_{h \cdot g \in h \cdot[A]\left(\begin{array}{l}k \\ i\end{array}\right)}\right)_{r}^{k}$, meaning that for every coloring $\Delta:\left\{\tilde{\mathscr{N}}_{g \in[A]\left(\begin{array}{l}k \\ (\end{array}\right)}\right\}_{k} \rightarrow r$ there exists $e_{j} \in \mathscr{E}$ such that $\left.\Delta\right\rceil e_{j}$. $\left\{\mathscr{M}_{h \cdot g \in h \cdot[A]\left(\begin{array}{c}k \\ i\end{array}\right)}\right\}_{k}$ is a constant coloring. Then for every coloring $\Delta:\left\{\mathscr{N}_{g \in[A]\left(\begin{array}{c}m \\ i\end{array}\right)}\right\}_{k} \rightarrow$ $r$ there exists $e_{j} \in \mathscr{E}$ such that $\left.\Delta\right\rceil\left\{\mathscr{M}_{h \cdot g \in[A]\left(\begin{array}{c}k \\ i\end{array}\right)}^{j}\right\}_{k}$ is a constant coloring.

PROOF. Obvious.

Recall that for $1 \leq i \leq k$ any $f \in\left\{\mathscr{M}_{g \in[A]\left(\begin{array}{c}m \\ i\end{array}\right)}\right\}_{k}$ is necessarily crossing, i.e., $f\rceil m \in[A]\left(\begin{array}{c}m \\ k\end{array}\right)$. For $i=0$ the situation is, obviously, different. Hence, in the 
following restrictedness property of the amalgamation we distinguish between $i=0$ and $i \geq 1$.

RESTRICTEDNESS PROPERTY. (1) Let $i \geq 1$ and let

$$
f \in\left\{\mathscr{N}_{g \in[A]\left(\begin{array}{c}
m \\
i
\end{array}\right)}\right\}_{i+1} \backslash\left\{\mathscr{N}_{h \cdot g \in h \cdot[A]\left(\begin{array}{c}
k \\
i
\end{array}\right)}\right\}_{i+1}
$$

Then there exists $j<z$ such that $f \in\left\{\mathscr{M}_{g \in[A]\left(\begin{array}{c}m \\ i\end{array}\right)}^{j}\right\}_{i+1}$.

(2) Let $i=0$ and $|A| \geq 2$. Also let $l+|A| \geq 3$, excluding the case $l=0$ and $|A|=2$.

Let $f \in\left\{\mathscr{N}_{g \in A^{m}}\right\}_{l+1} \backslash\left\{\mathscr{N}_{h \cdot g \in h \cdot A^{k}}\right\}_{l+1}$. Then there exists $j<z$ such that $f \in\left\{\mathscr{M}_{g \in A^{m}}^{j}\right\}_{l+1}$.

In human language: under certain conditions, the only $(l+1)$-spaces induced by $\mathscr{N}_{g \in[A]\left(\begin{array}{c}m \\ i\end{array}\right)}$ are those which are induced by one of the $\mathscr{M}_{g \in[A]}^{j}\left(\begin{array}{c}m \\ i\end{array}\right)$.

PROOF. Compare assertions (3) and (4) of Lemma 1.

We extend the amalgamation to families of $l$-spaces of $\mathscr{M}_{g \in[A]\left(\begin{array}{c}m \\ i\end{array}\right)} \subseteq A_{+}^{n}$. Let $\mathscr{G} \subseteq[A]\left(\begin{array}{c}m+n \\ l\end{array}\right)$ be a family of $l$-spaces of $\mathscr{M}_{g \in[A]\left(\begin{array}{c}m \\ i\end{array}\right)}$. For $j<z$ we denote by $\mathscr{G}^{j}$ the corresponding family of $l$-spaces in $\mathscr{M}_{g \in[A]\left(\begin{array}{c}m \\ i\end{array}\right)}^{j}$. By $\mathscr{G} *_{h} \mathscr{E}=\bigcup_{j<z} \mathscr{G}^{j}$ we denote the union of all these families.

CROSSING PROPERTY. If $\mathscr{G}$ is a family of crossing $l$-spaces then also $\mathscr{G} *_{h} \mathscr{E}$ is a family of crossing $l$-spaces.

PrOOF. Obvious.

GIRTH PROPERTY. Let $\mathscr{G}$ be a crossing family of $l$-spaces of $\mathscr{M}_{g \in[A]\left(\begin{array}{c}m \\ i\end{array}\right)}$. Then $k$-girth $\left(\mathscr{G} *_{h} \mathscr{E}\right) \geq\{k$-girth $\mathscr{E}, 2 \cdot(k$-girth $\mathscr{G})\}$.

REMARK. $k$-girth $\mathscr{E}$ is shorthand for the $k$-girth of the family of images of $\mathscr{M}_{h \cdot g \in h \cdot[A]\left(\begin{array}{c}k \\ i\end{array}\right)}$ with respect to the family $\mathscr{E}$. In particular,

$$
k \text {-girth } \mathscr{E}=k \text {-girth }\left\{\mathscr{M}_{h \cdot g \in h \cdot[A]\left(\begin{array}{c}
k \\
i
\end{array}\right)}^{j} \mid j<z\right\} .
$$

ProOF. Let $\mathscr{C}$ be a $k$-cycle in $\mathscr{G} *_{h} \mathscr{E}=\bigcup_{j<z} \mathscr{G}^{j}$. We view $\mathscr{C}$ as given by a set of $l$-spaces in $\mathscr{G} *_{h} \mathscr{E}$, where $|\mathscr{C}|$ is the length of the cycle. If $\mathscr{C} \subseteq \mathscr{G}^{j}$ for some $j<z$ then $|\mathscr{C}| \geq k$-girth $\mathscr{G}$. Otherwise, and as $\mathscr{G} *_{h} \mathscr{E}$ is crossing, the set of those $\mathscr{M}_{h \cdot g \in h \cdot[A]\left(\begin{array}{c}k \\ i\end{array}\right)}^{j}$ such that $f \cdot[A]\left(\begin{array}{l}l \\ k\end{array}\right) \cap\left\{\mathscr{M}_{h \cdot g \in h \cdot[A]\left(\begin{array}{c}k \\ i\end{array}\right)}^{j}\right\}_{k} \neq \varnothing$ for some $f \in \mathscr{C}$ contain a $k$-cycle $\mathscr{C}_{h}$. However, for each such $\mathscr{M}_{h \cdot g \in h \cdot[A]\left(\begin{array}{c}k \\ i\end{array}\right)}^{j} \in \mathscr{C}_{h}$ the original cycle $\mathscr{C}$ contains at least two distinct $f$ and $f^{\prime}$ with

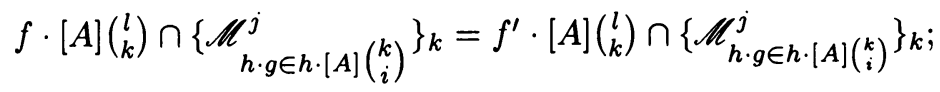

one to enter $\mathscr{M}_{h \cdot g \in h \cdot[A]\left(\begin{array}{c}k \\ i\end{array}\right)}^{j}$ and one to leave it. Hence, $|\mathscr{C}| \geq 2 \cdot(k$-girth $\mathscr{E})$.

8. Proof of the Sparse Graham-Rothschild Theorem. In this section we prove the Sparse Graham-Rothschild Theorem. For doing so we introduce some more notation and conventions. Let $i$ be a nonnegative integer, either 0 or 1 , depending on $A$. If $|A|=1$ then $i=1$ and $i=0$ otherwise. Let $a$ and $b$ be elements of $A$. If $|A|=1$ then, necessarily, $a=b$. Let $a \neq b$ for $|A| \geq 2$. Let 
$n$ be a positive integer satisfying $n \stackrel{[A]}{\longrightarrow}(m)_{r}^{k}$. Such an $n$ exists according to the Graham-Rothschild partition theorem for parameter sets.

By $\vec{a}$ we denote the sequence of length $n+2$ consisting just of $a$ 's, $\vec{a}=(a, \ldots, a)$. By $\vec{\lambda}=\left(\lambda_{0}, \ldots, \lambda_{n-1}\right)$ we denote the unique element in $[A]\left(\begin{array}{l}n \\ n\end{array}\right)$. For $j<z$ we denote by

$$
x_{j}^{z} \in[A]\left(\begin{array}{c}
z \cdot(n+2) \\
n
\end{array}\right)
$$

the sequence

$$
x_{j}^{z}=\underbrace{\vec{a} \otimes \cdots \otimes}_{j \text { times }} \vec{a} \otimes(a, b) \otimes \vec{\lambda} \otimes \underbrace{\vec{a} \otimes \cdots \otimes \vec{a}}_{(z-j-1) \text { times }} .
$$

For $h \in[A]\left(\begin{array}{l}n \\ k\end{array}\right)$ let $\tilde{h}$ be defined as in the proof of Lemma 1. More precisely, say, $h=\left(\alpha_{0}, \ldots, \alpha_{n-1}\right)$. Then $\tilde{h}=\left(\tilde{\alpha}_{0}, \ldots, \tilde{\alpha}_{n-1}\right)$, where $\tilde{\alpha}_{\nu}=\alpha_{\nu}$ for $\alpha_{\nu} \in A$ and $\tilde{\alpha}_{\nu}=\lambda_{j 0}$ for $\alpha_{\nu}=\lambda_{j}$, where $j_{0}=\min \left\{\nu<n \mid \alpha_{\nu}=\lambda_{j}\right\}$. For $j<z$ and $h \in[A]\left(\begin{array}{l}n \\ k\end{array}\right)$ let

$$
f_{j}^{h, z} \in[A]\left(\begin{array}{c}
(1+z) \cdot n \\
n
\end{array}\right)
$$

be defined by

$$
f_{j}^{h, z}=\vec{\lambda} \otimes \underbrace{\tilde{h} \otimes \cdots \otimes \tilde{h}}_{j \text { times }} \otimes \vec{\lambda} \otimes \underbrace{\tilde{h} \otimes \cdots \otimes \tilde{h}}_{(z-1-j) \text { times }}
$$

compare the proof of Lemma 1.

By $\tilde{\otimes}$ we denote the concatenation of parameter words with renumbering the parameters, this is something like a direct sum. More precisely, for $g \in[A]\left(\begin{array}{c}m \\ l\end{array}\right)$ and $h \in\left[A\left[\left(\begin{array}{c}m^{\prime} \\ l^{\prime}\end{array}\right)\right.\right.$ we have that

$$
g \tilde{\otimes} h \in[A]\left(\begin{array}{c}
m+m^{\prime} \\
l+l^{\prime}
\end{array}\right),
$$

namely, for $h=\left(\alpha_{0}, \ldots, \alpha_{m^{\prime}-1}\right)$ let $h^{+l}=\left(\alpha_{0}^{\prime}, \ldots, \alpha_{m^{\prime}-1}^{\prime}\right)$, where $\alpha_{\nu}^{\prime}=\alpha_{\nu}$ for $\alpha_{\nu} \in A$ and $\alpha_{\nu}^{\prime}=\lambda_{l+j}$ for $\alpha_{\nu}=\lambda_{j}$. We define $g \tilde{\otimes} h=g \otimes h^{+l}$.

By abuse of language we understand that

$$
\vec{\lambda} \tilde{\otimes} \vec{\lambda} \otimes \vec{\lambda}=\left(\lambda_{0} \cdots \lambda_{n-1}, \lambda_{n}, \ldots, \lambda_{2 n-1}, \lambda_{0} \cdots \lambda_{n-1}\right),
$$

i.e., the renumbering refers just to the sequence next to $\tilde{\otimes}$.

Let $y<z$ and let $Y \in[z]^{y}$ be a $y$-element subset of $\{0, \ldots, z-1\}$, say, $Y=$ $\left\{j_{0}, \ldots, j_{y-1}\right\}$ in increasing order. The parameter word

$$
x_{Y}^{z} \in[A]\left(\begin{array}{l}
z \cdot(n+2) \\
y \cdot(n+2)
\end{array}\right)
$$

is defined as

$$
x_{Y}^{z}=\underbrace{\vec{a} \tilde{\otimes} \ldots \tilde{\otimes} \vec{a}}_{j_{0} \text { times }} \tilde{\otimes} \vec{\lambda} \tilde{\otimes} \underbrace{\vec{a} \tilde{\otimes} \cdots \tilde{\otimes} \vec{a}}_{\left(j_{1}-j_{0}-1\right) \text { times }} \tilde{\otimes} \vec{\lambda} \tilde{\otimes} \cdots \tilde{\otimes} \vec{\lambda} \tilde{\otimes} \underbrace{\vec{a} \tilde{\otimes} \cdots \tilde{\otimes} \vec{a}}_{\left(z-j_{y-1}-1\right) \text { times }}
$$

where, here, $\vec{\lambda}=\left(\lambda_{0}, \ldots, \lambda_{n+1}\right)$.

OBSERVATION 1. $x_{Y}^{z} \cdot x_{\nu}^{y}=x_{j_{\nu}}^{z}$ for $\nu<y$.

PrOOF. Obvious.

Let $y<z$ and $Y \in[z]^{y}$ be as above and let $h \in[A]\left(\begin{array}{l}n \\ k\end{array}\right)$. The parameter word

$$
f_{Y}^{h, z} \in[A]\left(\begin{array}{c}
(1+z) \cdot n \\
(1+y) \cdot n
\end{array}\right)
$$


is defined as

$$
f_{Y}^{h, z}=\vec{\lambda} \otimes \underbrace{\tilde{h} \otimes \cdots \otimes \tilde{h}}_{j_{0} \text { times }} \tilde{\otimes} \vec{\lambda} \otimes \underbrace{\tilde{h} \otimes \cdots \otimes \tilde{h}}_{\left(j_{1}-j_{0}-1\right) \text { times }} \tilde{\otimes} \vec{\lambda} \otimes \ldots \tilde{\otimes} \vec{\lambda} \otimes \tilde{h} \otimes \underbrace{\tilde{h} \otimes \otimes \tilde{h}}_{\left(z-1-j_{y}-1\right) \text { times }} .
$$

OBSERVATION 2. $f_{Y}^{h, z} \cdot f_{\nu}^{h, y}=f_{j_{\nu}}^{h, z}$ for $\nu<y$.

PROOF. Obvious.

Let $\left(f_{\nu}\right)_{\nu<n_{0}^{\circ}}$ be an enumeration of $[A]\left(\begin{array}{l}n \\ m\end{array}\right)$. We define an $n$-partite configuration $\mathscr{M}_{g \in[A]\left(\begin{array}{c}n \\ i\end{array}\right)}^{0} \subseteq\left\{x_{\nu}^{n_{0}^{0}} \cdot g \mid \nu<n_{0}^{0}, g \in[A]\left(\begin{array}{c}n \\ i\end{array}\right)\right\}$ by

$$
\mathscr{M}_{g}^{0}=\left\{x_{\nu}^{n_{0}^{0}} \cdot g \mid \nu<n_{0}^{0} \text { and } g \in f_{\nu} \cdot[A]\left(\begin{array}{c}
m \\
i
\end{array}\right)\right\} .
$$

OBSERVATION 3 .

$$
\left\{\mathscr{M}_{g \in[A]\left(\begin{array}{c}
n \\
i
\end{array}\right)}^{0}\right\}_{k} \subseteq\left\{f \otimes x_{\nu}^{n_{0}^{0}} \cdot f \mid \nu<n_{0}^{0}, f \in[A]\left(\begin{array}{l}
n \\
k
\end{array}\right)\right\}
$$

and

$$
\left\{\mathscr{M}_{g \in[A]\left(\begin{array}{c}
n \\
m
\end{array}\right)}^{0}\right\}_{m}=\left\{f_{\nu} \otimes x_{\nu}^{n_{0}^{0}} \cdot f_{\nu} \mid \nu<n_{0}^{0}\right\}
$$

In other words, all $k$, resp., $m$-subspaces are crossing.

PROOF. For $\nu<\nu^{\prime}$ and $g, g^{\prime} \in[A]\left(\begin{array}{c}n \\ i\end{array}\right)$ the parameter words $x_{\nu}^{n_{0}^{0}} \cdot g$ and $x_{\nu^{\prime}}^{n_{0}^{0}} \cdot g^{\prime}$ generate a subspace which is not induced by $\left\{x_{\nu}^{n_{0}^{0}} \cdot g \mid \nu<n_{0}^{0}, g \in[A]\left(\begin{array}{c}n \\ i\end{array}\right)\right\}$.

The crucial property of the $n$-partite $i$-configuration $\mathscr{M}_{g \in[A]\left(\begin{array}{l}n \\ i\end{array}\right)}^{0}$ is given in the next observation.

OBSERVATION 4. Let $\Delta:\left\{\mathscr{M}_{g \in[A]\left(\begin{array}{c}n \\ i\end{array}\right)}^{0}\right\}_{k} \rightarrow r$ be such that $\Delta(g)=\Delta(\tilde{g})$ for all $g$ and $\tilde{g}$ in $\left\{\mathscr{M}_{g \in[A]\left(\begin{array}{c}n \\ i\end{array}\right)}^{0}\right\}_{k}$ with $\left.\left.g\right\rceil n=\tilde{g}\right\rceil n$. Then there exists $f \in\left\{\mathscr{M}_{g \in[A]\left(\begin{array}{c}n \\ i\end{array}\right)}^{0}\right\}_{m}$ which is monochromatic, i.e., $\Delta\rceil f \cdot[A]\left(\begin{array}{c}m \\ k\end{array}\right)$ is a constant coloring.

ProOF. Such a coloring $\Delta$ induces a coloring $\Delta^{*}:[A]\left(\begin{array}{l}n \\ k\end{array}\right) \rightarrow r$ by $\left.\Delta^{*}(g\rceil n\right)=$ $\Delta(g)$ for any $g \in\left\{\mathscr{M}_{g \in[A]\left(\begin{array}{c}n \\ i\end{array}\right)}\right\}_{k}$. By choice of $n$ there exists $j<z$ such that $f_{j}$ is monochromatic with respect to $\Delta^{*}$. Then $f_{j} \otimes x_{j}^{n_{0}^{0}} \cdot f_{j}$ has the desired properties.

Let $\left(h_{p}\right)_{p<q}$ be an enumeration of $[A]\left(\begin{array}{l}n \\ k\end{array}\right)$ and assume by induction that the $n$-partite configuration $\mathscr{M}_{g \in[A]\left(\begin{array}{l}n \\ i\end{array}\right)}^{p}$ has been constructed such that

(1) $\mathscr{M}_{g}^{p} \subseteq\left(\left\{x_{\nu}^{n_{0}^{p}} \mid \nu<n_{0}^{p}\right\} \otimes\left\{f_{\nu}^{h_{0}, n_{1}^{p}} \mid \nu<n_{1}^{p}\right\} \otimes \cdots \otimes\left\{f_{\nu}^{h_{p-1}, n_{p}^{p}} \mid \nu<n_{p}^{p}\right\}\right) \cdot g$.

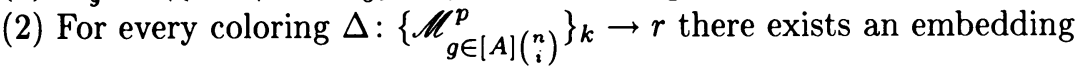

$$
f: \mathscr{M}_{g \in[A]\left(\begin{array}{c}
n \\
i
\end{array}\right)}^{0} \hookrightarrow \mathscr{M}_{g \in[A]\left(\begin{array}{c}
n \\
i
\end{array}\right)}^{p}
$$

such that $\Delta(f \cdot g)=\Delta\left(f \cdot g^{\prime}\right)$ for all $g, g^{\prime} \in\left\{\mathscr{M}_{g \in[A]\left(\begin{array}{c}n \\ i\end{array}\right)}^{0}\right\}_{k}$ with $\left.\left.g\right\rceil n=g^{\prime}\right\rceil n=h_{\mu}$ for some $\mu<p$.

(3) $k$-girth $\left\{\mathscr{M}_{g \in[A]\left(\begin{array}{c}n \\ i\end{array}\right)}^{p}\right\}_{m}>c$.

Note that (2) holds vacuously for $\mathscr{M}_{g \in[A]\left(\begin{array}{c}n \\ i\end{array}\right)}^{0}$ and (3) is also satisfied as, by Observation 3 , any two distinct $m$-spaces in $\left\{\mathscr{M}_{g \in[A]\left(\begin{array}{c}n \\ i\end{array}\right)}^{0}\right\}_{m}$ do not even have a common $i$-space. (1) is obvious. 
We show how to construct $\mathscr{M}_{g \in[A]\left(\begin{array}{l}n \\ i\end{array}\right)}^{p+1}$ such that it satisfies again (1), (2) and (3). Consider $h_{p} \in[A]\left(\begin{array}{l}n \\ k\end{array}\right)$. We define a $(p+1)$-partite graph $G: \prod_{\mu \leq p} n_{\mu}^{p} \rightarrow \mathscr{P}\left([A]\left(\begin{array}{l}k \\ i\end{array}\right)\right)$ $\left(\mathscr{P}\left([A]\left(\begin{array}{l}k \\ i\end{array}\right)\right)\right.$ denotes the power set of $\left.[A]\left(\begin{array}{l}k \\ i\end{array}\right)\right)$ by

$$
G\left(\nu_{0}, \ldots, \nu_{p}\right)=\left\{g \in[A]\left(\begin{array}{l}
k \\
i
\end{array}\right) \mid\left(x_{\nu_{0}}^{n_{0}^{p}} \otimes f_{\nu_{1}}^{h_{0}, n_{1}^{p}} \otimes \cdots \otimes f_{\nu_{p}}^{h_{p-1}, n_{p}^{p}}\right) \cdot\left(h_{p} \cdot g\right) \in \mathcal{N}_{h_{p}}^{p}\right\} .
$$

Note that $\mathscr{P}\left([A]\left(\begin{array}{l}k \\ i\end{array}\right)\right)$, the set of subsets of $[A]\left(\begin{array}{l}k \\ i\end{array}\right)$ can be viewed as $\left\{[A]\left(\begin{array}{l}k \\ i\end{array}\right) \rightarrow 2\right\}$.

For $g \in[A]\left(\begin{array}{l}k \\ i\end{array}\right)$ the set $I(g) \subseteq\{0, \ldots, p\}$ is the complement of the set of those $1 \leq \mu \leq p$ such that $h_{p} \cdot g \in h_{\mu-1} \cdot[A]\left(\begin{array}{l}k \\ i\end{array}\right)$. By the sparse product lemma there exists a $(p+1)$-partite graph $\tilde{G}: \prod_{\mu \leq p} n_{\mu}^{p+1} \rightarrow \mathscr{P}\left([A]\left(\begin{array}{l}k \\ i\end{array}\right)\right)$ such that $I(g)=\tilde{I}(g)$ for all $g \in[A]\left(\begin{array}{c}k \\ i\end{array}\right)$ and there exists a family

$$
\mathscr{S}=\left\{Y_{0}^{\nu} \times \cdots \times Y_{p}^{\nu} \in\left[n_{0}^{p+1}\right]^{n_{0}^{p}} \times \cdots \times\left[n_{p}^{p+1}\right]_{p}^{n_{p}^{p}} \mid \nu<n_{p+1}^{p+1}\right\}
$$

of $G$-subgraphs of $\tilde{G}$ such that $I(g)=\tilde{I}(g)$ for every $g \in[A]\left(\begin{array}{l}k \\ i\end{array}\right)$, girth $\mathscr{S}>c$ and $(\tilde{G}, \mathscr{S}) \rightarrow(G)_{r}^{[A]\left(\begin{array}{c}k \\ i\end{array}\right)}$. Observe that the $k$-partite $i$-configuration $\mathscr{M}_{h_{p} \cdot g \in h_{p} \cdot[A]\left(\begin{array}{l}k \\ i\end{array}\right)}^{p}$ can be described in terms of $G$ as

$$
\mathscr{M}_{h_{p} \cdot g}=\left\{\left(x_{\nu_{0}}^{n_{0}^{p}} \otimes f_{\nu_{1}}^{h_{0}, n_{1}^{p}} \otimes \cdots \otimes f_{\nu_{p}}^{h_{p-1}, n_{p}^{p}}\right) \cdot h_{p} \cdot g \mid\left(\nu_{0}, \ldots, \nu_{p}\right) \in \prod_{\mu \leq p} n_{\mu}^{p}\right.
$$

$$
\text { and } \left.g \in G\left(\nu_{0}, \ldots, \nu_{p}\right)\right\} \text {. }
$$

In particular,

\section{OBSERVATION 5 .}

$$
\begin{aligned}
& \left\{\mathscr{M}_{\left.h_{p} \cdot g \in h_{p} \cdot[A]\left(\begin{array}{c}
k \\
i
\end{array}\right)\right\}_{k}}\right. \\
& =\left\{\left(x_{\nu_{0}}^{n_{p}^{p}} \times f_{\nu_{1}}^{h_{0}, n_{1}^{p}} \otimes \cdots \otimes f_{\nu_{p}}^{h_{p-1}, n_{p}^{p}}\right) \cdot h_{p} \mid\left(\nu_{0}, \ldots, \nu_{p}\right) \in \prod_{\mu \leq p} n_{\mu}^{p}\right. \\
& \left.\quad \text { and } G\left(\nu_{0}, \ldots, \nu_{p}\right)=[A]\left(\begin{array}{c}
k \\
i
\end{array}\right)\right\} .
\end{aligned}
$$

ProOF. Obvious.

We define a $k$-partite $i$-configuration $\tilde{\mathscr{N}}_{g \in[A]\left(\begin{array}{l}k \\ i\end{array}\right)}$ by

$$
\begin{aligned}
& \tilde{\mathscr{N}_{g}}=\left\{\left(x_{\nu_{0}^{0}}^{n_{0}^{p+1}} \otimes f_{\nu_{1}}^{h_{0}, n_{1}^{p+1}} \otimes \cdots \otimes f_{\nu_{p}}^{h_{p-1}, n_{p}^{p+1}}\right) \cdot h_{p} \cdot g \mid\left(\nu_{0}, \ldots, \nu_{p}\right) \in \prod_{\mu \leq p} n_{\nu}^{p+1}\right. \\
& \text { and } \left.g \in \tilde{G}\left(\nu_{0}, \ldots, \nu_{p}\right)\right\} \text {. }
\end{aligned}
$$

Observe that this is a proper definition as $I(g)=\tilde{I}(g)$ for all $g \in[A]\left(\begin{array}{l}k \\ i\end{array}\right)$. For each $\nu<n_{p+1}^{p+1}$ define the embedding

$$
e_{\nu}: \mathscr{M}_{h_{p} \cdot g \in h_{p} \cdot[A]\left(\begin{array}{c}
k \\
i
\end{array}\right)}^{p} \hookrightarrow \tilde{\mathscr{N}}_{g \in[A]\left(\begin{array}{l}
k \\
i
\end{array}\right)}
$$


by

$$
e_{\nu}=\left(\lambda_{0}, \ldots, \lambda_{k-1}\right) \tilde{\otimes}\left(x_{Y_{0}^{\nu}}^{n_{0}^{p+1}} \tilde{\otimes} f_{Y_{1}^{\nu}}^{h_{0}, n_{1}^{p+1}} \tilde{\otimes} \cdots \tilde{\otimes} f_{Y_{p}^{\nu}}^{h_{p-1}, n_{p}^{p+1}}\right) .
$$

Let $\mathscr{E}=\left\{e_{\nu} \mid \nu<n_{p+1}^{p+1}\right\}$.

OBSERVATION 6 . Each $e_{\nu}$ actually is an embedding. Moreover, $k$-girth $\mathscr{E}>c$.

PROOF. That $e_{\nu}$ is an embedding follows from Observations 1 and 2. That $k$-girth $\mathscr{E}>c$ follows, then, as

$$
\operatorname{girth}\left\{Y_{0}^{\nu} \times \cdots \times Y_{p}^{\nu} \mid \nu<n_{p+1}^{p+1}\right\}>c .
$$

OBSERVATION 7. $\left(\tilde{\mathscr{N}}_{g \in[A]\left(\begin{array}{l}k \\ i\end{array}\right)}, \mathscr{E}\right) \rightarrow\left(\mathscr{M}_{h_{p} \cdot g \in h_{p} \cdot[A]\left(\begin{array}{l}k \\ i\end{array}\right)}^{p}\right)_{r}^{k}$.

ProOF. Observations 5 and 6 as well as the fact that $(\tilde{\mathscr{G}}, \mathscr{S}) \rightarrow(\mathscr{G})_{r}^{[A]\left(\begin{array}{l}k \\ i\end{array}\right)}$.

We now define

$$
\mathscr{M}_{g \in[A]\left(\begin{array}{l}
n \\
i
\end{array}\right)}^{p+1}=\mathscr{M}_{g \in[A]\left(\begin{array}{l}
n \\
i
\end{array}\right)}^{p} *_{h_{p}}\left(\tilde{\mathscr{N}}_{g \in[A]\left(\begin{array}{l}
k \\
i
\end{array}\right)}, \mathscr{E}\right) .
$$

OBSERVATION 8.

$$
\left\{\mathscr{M}_{g \in[A]\left(\begin{array}{c}
n \\
i
\end{array}\right)}^{p+1}\right\}_{m}=\left\{\mathscr{M}_{g \in[A]\left(\begin{array}{c}
n \\
i
\end{array}\right)}^{p}\right\}_{m} *_{h_{p}}\left(\tilde{\mathscr{N}}_{g \in[A]\left(\begin{array}{c}
k \\
i
\end{array}\right)}, \mathscr{E}\right) .
$$

PROOF. This follows immediately from the restrictedness property of the amalgamation.

OBSERVATION 9. $\mathscr{M}_{g \in[A]\left(\begin{array}{l}n \\ i\end{array}\right)}^{p+1}$ again satisfies (1), (2) and (3).

PROOF. The first assertion is straightforwardly verified looking at the definition of the amalgamation. (2) and (3), then, follow from the coloring property together with Observation 7 and the girth property of the amalgamation together with Observation 8.

Finally, by Observation 4, the $n$-partite $i$-configuration $\mathscr{M}_{g \in[A]\left(\begin{array}{l}n \\ i\end{array}\right)}^{q}$ has the desired properties, i.e., let

$$
\mathscr{F}=\bigcup_{g \in[A]\left(\begin{array}{l}
n \\
i
\end{array}\right)} g \otimes \mathscr{M}_{g}^{q} .
$$

This completes the proof of the Sparse Graham-Rothschild Theorem.

9. Applications and corollaries. In this section we give some applications and corollaries of the Sparse Graham-Rothschild Theorem.

9.1 Sparse van der Waerden sets. In 1926 B. L. van der Waerden [1927] proved a theorem which nowadays is known as van der Waerden's theorem on arithmetic progressions:

For every partition of the nonnegative integers into finitely many classes one of these classes contains arithmetic progressions of arbitrary finite length.

Let us call a set $S$ of positive integers a $k$-van der Waerden set if for every partition of $S$ into finitely many classes one of the classes contains an arithmetic progression of length $k$. Clearly, such sets $S$ contain a lot of $k$-element arithmetic progressions. J. Spencer [1975] and, independently, J. Nešetrril and V. Rödl [1976] showed that there exist $k$-van der Waerden sets not containing any $(k+1)$-element arithmetic progression. They, then, raised the question whether there exist $k$-van 
der Waerden sets $S$ such that the family of $k$-element arithmetic progressions does not form short cycles, i.e., $S$ should be sparse.

(We say that a family of finite subsets $A_{0}, \ldots, A_{c-1}$ of nonnegative integers contains a cycle of length at most $c$ if $\left|\bigcup_{i<c} A_{i}\right| \leq\left(\sum_{i<c}\left|A_{i}\right|\right)-c$, so a cycle of length two is given by two sets $A$ and $B$ intersecting in at least two vertices. The reader should convince himself that this definition renders precise what one intuitively would call a cycle.)

We answer this question in the affirmative:

For all positive integers $k \geq 3$ and $c$ there exists a $k$-van der Waerden set such that the family of its $k$-element arithmetic progressions does not contain a cycle of length at most $c$.

For short: there exist sparse $k$-van der Waerden sets.

The existence of sparse van der Waerden sets follows from a slight strengthening of the Sparse Graham-Rothschild Theorem which is explained in the next subsection. So we postpone the proof until §9.3. Sparse van der Waerden sets have been also constructed, independently and using a different approach, by V. Rödl [1986].

9.2 A sparse version of the Hales-Jewett theorem. Years after van der Waerden obtained his 'Beweis einer Baudetschen Vermutung', A. W. Hales and R. I. Jewett revealed the combinatorial part of van der Waerden's theorem. The Hales-Jewett theorem [1963] is concerned with colorings of $A^{n}$, asserting the existence of a monochromatic $m$-space.

Thus for $k=0$ the Sparse Graham-Rothschild Theorem actually is a sparse version of the Hales-Jewett theorem. However, the same proof establishes, for $k=0$, a somewhat stronger result.

Let us denote by $\mathscr{S}_{A}$ the symmetric group on $A$, i.e., $\mathscr{S}_{A}$ is the set of all permutations $\pi: A \rightarrow A$. By $\varepsilon$ we denote the identity.

DEFINITION. For nonnegative integers $m \leq n$ we denote by $[\tilde{A}]\left(\begin{array}{l}n \\ m\end{array}\right)$ the set of mappings $f: n \rightarrow A \cup\left(\left\{\lambda_{0}, \ldots, \lambda_{m-1}\right\} \times \mathscr{S}_{A}\right)$ satisfying

for every $j<m$ there exists $i<n$ with $f(i)=\left(\lambda_{j}, \varepsilon\right)$, moreover, $f\left(\min f^{-1}\left(\left\{\lambda_{j}\right\} \times \mathscr{S}_{A}\right)\right)=\left(\lambda_{j}, \varepsilon\right)$ and $\min f^{-1}\left(\left\{\lambda_{i}\right\} \times \mathscr{S}_{A}\right)<$ $\min f^{-1}\left(\left\{\lambda_{j}\right\} \times \mathscr{S}_{A}\right)$ for all $i<j<m$.

For $f \in[\tilde{A}]\left(\begin{array}{c}n \\ m\end{array}\right)$ and $g \in A^{m}\left(=[A]\left(\begin{array}{c}m \\ 0\end{array}\right)\right)$ the composition $f \cdot g \in A^{n}$ is defined by $(f \cdot g)(i)=f(i)$ if $f(i) \in A$ and $(f \cdot g)(i)=\pi(g(j))$ if $f(i)=\left(\lambda_{j}, \pi\right)$. As before $M=f \cdot A^{m}$ is the parameter set described by $f$.

REMARK. Actually, this generalized concept of parameter sets is the original concept of R. L. Graham and B. L. Rothschild [1971].

A subset $\mathscr{M} \subseteq A^{m}$ is a configuration. An embedding of a configuration $\mathscr{M} \subseteq A^{m}$ into a configuration $\mathscr{N} \subseteq A^{n}$ is given by an $f \in[\tilde{A}]\left(\begin{array}{l}n \\ m\end{array}\right)$ such that $f \cdot \mathscr{M} \subseteq \mathscr{N}$. We write shorthand $f: \mathscr{M} \hookrightarrow \mathscr{N}$.

Embeddings $f \in[A]\left(\begin{array}{l}n \\ m\end{array}\right)$, i.e., embeddings which are given by parameter words in the sense of $\S 2$, are special embeddings.

The image $f \cdot \mathscr{M}$ is an $\mathscr{M}$-subconfiguration of $\mathscr{N}$. The binomial coefficient $\left(\begin{array}{l}\mathcal{N} \\ \mathscr{M}\end{array}\right)$ denotes the set of $\mathscr{M}$-subconfigurations of $\mathscr{N}$.

A cycle of length $c$ in $\mathscr{E} \subseteq\left(\begin{array}{l}\mathscr{N} \\ \mathscr{M}\end{array}\right)$ is given by $c$ mutually distinct $\mathscr{M}$-subconfigurations $\mathscr{M}_{0}, \mathscr{M}_{1}, \ldots, \mathscr{M}_{c-1} \in \mathscr{E}$ and $c$ mutually distinct vertices $x_{0}, \ldots, x_{c-1} \in \mathscr{N}$ 
such that $x_{0} \in \mathscr{M}_{0} \cap \mathscr{M}_{1}, x_{1} \in \mathscr{M}_{1} \cap \mathscr{M}_{2}, \ldots, x_{c-1} \in \mathscr{M}_{c-1} \cap \mathscr{M}_{0}$. The length of the shortest cycle in $\mathscr{E}$ is denoted by girth $\mathscr{E}$.

A SPARSE HALES-JEWETT ThEOREM. Let $A$ be a finite set having at least three elements. Let $m, r$ and $c$ be positive integers. Then there exists a positive integer $n$ and there exists a configuration $\mathscr{N} \subseteq A^{\prime}$ such that

(1) $\operatorname{girth}\left(\begin{array}{c}\mathscr{N} \\ A^{m}\end{array}\right)>c$,

(2) for every coloring $\Delta: \mathscr{N} \rightarrow r$ there exists a special embedding $f: A^{m} \hookrightarrow \mathscr{N}$ such that $\Delta\rceil f \cdot A^{m}$ is a constant coloring.

REMARK. Observe that (1) asserts that the family of $A^{m}$-subconfigurations of $\mathscr{N}$ (even in the generalized sense) does not contain short cycles, while (2) shows that a monochromatic $A^{m}$-subconfiguration can be obtained via a special embedding.

PROOF. An inspection of the proof of the Sparse Graham-Rothschild Theorem for $k=0$ shows that the same proof actually establishes the seemingly stronger statement of the Sparse Hales-Jewett Theorem. The reason is that also a 1-space in the generalized sense is determined by two of its vertices.

9.3 Proof of 'there exist sparse k-van der Waerden sets'. It is well known that it suffices to prove the following result:

THEOREM. Let $k>3, r$ and $c$ be positive integers. Then there exists a finite set $S$ of positive integers such that

(1) the family of arithmetic progressions of length $k$ in $S$ does not contain a cycle of less than $c$,

(2) for every coloring $\Delta: S \rightarrow r$ there exists a monochromatic $k$-element arithmetic progression.

PROOF. Let $A=\{0, \ldots, k-1\}$ and let $\mathscr{N} \subseteq A^{n}$ be according to the Sparse Hales-Jewett Theorem with respect to $r$ and $c$ and $m=1$. Let $p$ be a prime number which is sufficiently large, $p>k+k^{2}$ suffices. Consider the mapping $\varphi: A^{n} \rightarrow \mathbf{N}$ which is given by $\varphi\left(\alpha_{0}, \ldots, \alpha_{n-1}\right)=\sum_{i<n} \alpha_{i} \cdot p^{i}$.

We claim that the set $S=\varphi(\mathscr{N})$ has the desired properties. As, trivially, the image under $\varphi$ of a 1-space $f \in[A]\left(\begin{array}{l}n \\ 1\end{array}\right)$ is an arithmetic progression it remains to verify (1). We show that preimages of arithmetic progressions in $\varphi(\mathscr{N})$ are 1-spaces in the generalized sense in $\mathscr{N}$. This follows immediately from the next lemma.

LEMMA. Let $k$ be a positive integer and let $p$ be a sufficiently large prime number, viz. $k^{2}+k<p$. Let $S_{n}=\left\{\sum_{i<n} \alpha_{i} \cdot p^{i} \mid 0 \leq \alpha_{i}<k\right\}$. Let $a=\sum_{i<n} \alpha_{i} \cdot p^{i} \in S_{n}$ and let $b=\sum_{i<n} \beta_{i} \cdot p^{i}$, where $0 \leq \beta_{i}<p$, be such that $a+s \cdot b \in S_{n}$ for $0 \leq s<k$, i.e., $\{a+s \cdot b \mid s<k\}$ is an arithmetic progression of length $k$ in $S_{n}$. Then the number $b$ has the following special structure: Let $\beta_{-1}=0$; if $\beta_{i-1} \in\{p-1, p-2\}$ then $\beta_{i} \in\{0, p-1, p-2\}$; if $\beta_{i} \in\{0,1\}$ then $\beta_{i+1} \in\{0,1, p-1\} ; \beta_{n-1} \in\{0,1\}$. Moreover, if $\beta_{i}=1$ then $\alpha_{i}=0$; if $\beta_{i}=p-2$ then $\alpha_{i}=p-1$; if $\beta_{i-1} \in\{0,1\}$ and $\beta_{i}=p-1$ then $\alpha_{i}=p-1$; if $\beta_{i-1} \in\{p-1, p-2\}$ and $\beta_{i}=0$ then $\alpha_{i}=0$.

PROOF. Say $a+s \cdot b=\sum_{i<n} \gamma_{s, i} \cdot p^{i}$, where $0 \leq \gamma_{s, i}<k$. Now we proceed by induction on $i$. If $\beta_{i-1} \in\{0,1\}$ then, by induction, no overflow occurs into the $i$ th position, i.e., $\gamma_{s, i} \equiv\left(\alpha_{i}+s \cdot \beta_{i}\right) \bmod p$ for $0 \leq s<k$. As $p$ is sufficiently large we conclude that $\beta_{i} \equiv 0$ or $\beta_{i} \equiv 1$ or $\beta_{i} \equiv-1 \bmod p$, i.e., $\beta_{i} \in\{0,1, p-1\}$. Moreover, if $\beta_{i}=1$ then necessarily $\alpha_{i}=0$ and if $\beta_{i}=p-1$ then necessarily 
$\alpha_{i}=k-1$. Note, then, that $\beta_{i}=p-1$ produces an overflow into position $i+1$ for each $0 \leq s<k$. If $\beta_{i-1} \in\{p-1, p-2\}$ then, again by induction, an overflow occurs, i.e., $\gamma_{s, i} \equiv\left(\alpha_{i}+s \cdot \beta_{i}+s\right) \bmod p_{i}$. By the same argument as before we see that $\beta_{i} \in\{0,1, p-1\}$, i.e., $\beta_{i} \in\{p-2, p-1,0\}$. If $\beta_{i}=0$ then $\alpha_{i}=0$ and if $\beta_{i}=p-2$ then $\alpha_{i}=k-1$.

9.4 A sparse finite union theorem. The particular case $A=\{0\}$ and $k=1$ of the Sparse Graham-Rothschild Theorem deserves special attention. Using the interpretation given in $\S 2$ for $A=\{0\}$ we immediately obtain

A SPARSE FINITE UNION THEOREM. Let $m, r$ and $c$ be positive integers. Then there exists a finite family $\mathscr{S}$ of nonempty finite sets such that

(1) The set system $\{\mathscr{S}\}_{m}$ having $\mathscr{S}$ as set of vertices and edges $\left\{\bigcup_{i \in I} S_{i} \mid I\right.$ is a nonempty subset of $\{0, \ldots, m-1\}\}$ for each choice of pairwise disjoint sets $S_{0}, \ldots$, $S_{m-1} \in \mathscr{S}$ such that also all unions $\bigcup_{i \in I} S_{i}$ belong to $\mathscr{S}$ has girth larger than $c$.

(2) For every $r$-coloring $\Delta: \mathscr{S} \rightarrow r$ there exist $m$ pairwise disjoint sets $S_{0}, \ldots$, $S_{m-1} \in \mathscr{S}$ such that for all nonempty $I \subseteq\{0, \ldots, m-1\}$ the finite union $\bigcup_{i \in I} S_{i}$ belongs to $\mathscr{S}$ and all these finite unions are colored the same.

REMARKS. (1) For $c=1$, i.e., without any restriction on cycles, the corresponding result is known as the Rado-Folkman-Sanders theorem, compare Graham, Rothschild and Spencer [1980].

(2) For $c \geq 2$ it particularly follows that $\mathscr{S}$ does not contain $(m+1)$-mutually disjoint sets together with all their unions. This restricted result has been proved in Nešetřil and Rödl [1986].

9.5 Sparse k-Rado sets. I. Schur [1917] showed that for every partition of the nonnegative integers into finitely many classes there exist integers $x, y$ and $x+$ $y$ in one of these classes. Schur's investigations were motivated by attempts in connection with Fermat's last theorem. It has been said that also the motivation for van der Waerden's theorem on arithmetic progressions was coming originally from the Berlin circle around Schur, cf. Brauer [1973].

In his celebrated thesis, then, which was written in Berlin under the supervision of Schur, R. Rado [1933] gave a complete and satisfactory explanation of the common background of Schur's and van der Waerden's results, compare also Deuber and Voigt [1983] or Graham, Rothschild and Spencer [1980].

In particular, from Rado's investigations a generalization of Schur's result, known as Rado's finite sum theorem follows immediately:

For every partition of the positive integers into finitely many classes and for every positive integer $k$ there exist integers $x_{0}, \ldots, x_{k-1}$ in one of these classes such that for all nonempty $I \subseteq\{0, \ldots, k-1\}$ also the finite sum $\sum_{i \in I} x_{i}$ belongs to this same class.

Nowadays it is well known that Rado's finite sum theorem can be deduced easily from van der Waerden's theorem on arithmetic progressions and we do not hesitate to repeat the argument, for another short proof see Nešetrril and Rödl [1983].

We show by induction on $\left(k_{0}, \ldots, k_{r-1}\right)$ that for every partition $\mathbf{N}=\mathscr{A}_{0} \cup \ldots \cup$ $\mathscr{A}_{r-1}$ there exists an $i<r$ such that class $\mathscr{A}_{i}$ contains $k_{i}$ integers and all their finite sums. By van der Waerden's theorem we know that one of the $\mathscr{A}_{i}$ contains arbitrary long arithmetic progressions. Say, $\mathscr{A}_{0}$ contains $\{a+l \cdot d \mid 1 \leq l \leq N\}$, 
where $N$ is sufficiently large. Consider $\{l \cdot d \mid 1 \leq l<N\}$. By induction, there exists $1 \leq i<r$ such that $\mathscr{A}_{i} \cap\{l \cdot d \mid 1 \leq l<N\}$ contains $k_{i}$ integers and all their finite sums or, otherwise, $\mathscr{A}_{0} \cap\{l \cdot d \mid 1 \leq l<N\}$ contains $k_{0}-1$ integers and all their finite sums. But then we may as well add $a$, the first element of the long arithmetic progression in $\mathscr{A}_{0}$, to find $k_{0}$ integers as desired.

Now let us call a set $S$ of positive integers an $m$-Rado set if for every partition of $S$ into finitely many classes one of the classes contains $m$ integers together with all their sums (without repetition, as before).

In view of Schur's result it is justified to call 2-Rado sets also Schur sets. J. Nešetril and V. Rödl [1986a] showed that sparse Schur sets exist. Their proof is based on the fact that Schur's theorem can be deduced from Ramsey's theorem for pairs. Additionally, the existence of sparse Ramsey graphs is invoked.

Nešetriil and Rödl, then, raised the question whether sparse $m$-Rado sets exist for arbitrary $m$.

We answer this question in the affirmative.

Notation. If $S$ is a set of positive integers we denote by $\{S\}_{m}$ the set system having $S$ as set of vertices and edges $\left\{\sum_{i \in I} x_{i} \mid I\right.$ is a nonempty subset of $\{0, \ldots, m-$ $1\}\}$ for each choice of integers $x_{0}, \ldots, x_{m-1} \in S$ such that also all sums $\sum_{i \in I} x_{i}$ belong to $S$.

Sparse $m$-Rado sets exist. For all positive integers $m$ and $c$ there exists an $m$ Rado set $S$ such that $\{S\}_{m}$ does not contain cycles of length at most $c$.

REMARK. In particular, for $c \geq 2$, such an $S$ does not contain $(m+1)$ integers and all their sums. So this strengthens the result from Nešetril and Rödl [1986b].

The existence of sparse $m$-Rado sets follows immediately from the following theorem.

THEOREM. Let $m, r$ and $c$ be positive integers. Then there exists a finite set $S$ of positive integers such that $\operatorname{girth}\{S\}_{m}>c$ but for every $r$-coloring $\Delta: S \rightarrow r$ there exists a set $X \in\{S\}_{m}$ such that $\left.\Delta\right\rceil X$ is a constant coloring.

Proof. Let $\mathscr{S}$ be a finite family of finite and nonempty sets satisfying (1) and (2) of the Sparse Finite Union Theorem (with respect to $m, r$ and $c$ ). Without loss of generality the elements of $\mathscr{S}$ are sets of positive integers. To each $Z \in \mathscr{S}$ we associate the positive integer $n(Z)=\sum_{i \in Z} 3^{i}$ and let $S=\{n(Z) \mid Z \in \mathscr{S}\}$. Then, for $Z$ and $Z^{\prime}$ disjoint elements of $\mathscr{S}, n(Z)+n\left(Z^{\prime}\right) \in S$ if and only if $Z \cup Z^{\prime} \in \mathscr{S}$ and, thus, $S$ has the desired properties.

10. Graphs over parameter sets. Let $\alpha=\left(\alpha_{0}, \ldots, \alpha_{a}\right)$ be a sequence of positive integers. An $\alpha$-graph on $A^{m}$ is given by a mapping $\mathscr{E}:[A]\left(\begin{array}{c}m \\ i\end{array}\right) \rightarrow \alpha_{i}$ for $0 \leq i \leq a$ (by abuse of language we use the same $\mathscr{E}$ for all $i$ ).

Whenever possible we simply say that $\mathscr{E}$ is an $\alpha$-graph, resp., we write $(m, \mathscr{E})$ to indicate that $\mathscr{E}$ is an $\alpha$-graph on $A^{m}$. The intended interpretation is that $A^{m}$ is the set of vertices of the $\alpha$-graph $\mathscr{E}$ and the mapping $\mathscr{E}$ indicates for each $i$-parameter word $g \in[A]\left(\begin{array}{c}m \\ i\end{array}\right)$ which kind of edge it forms. The numbers $\mathscr{E}(g)$ can also be interpreted as multiplicities, so $\mathscr{E}(g)=0$ indicates the absence of the corresponding edge $g$. For $|A|=1$ vertices should be interpreted as nonempty subsets of $\{0, \ldots, n-1\}$, cf. our remark in $\S 2$. 
The notion of embeddings between $\alpha$-graphs is defined straightforwardly. Let $(m, \mathscr{E})$, resp. $(\tilde{m}, \tilde{\mathscr{E}})$, be $\alpha$-graphs. An embedding $f: \mathscr{E} \hookrightarrow \tilde{\mathscr{E}}$ is given by an $f \in[A]\left(\begin{array}{c}\tilde{m} \\ m\end{array}\right)$ such that $\mathscr{E}(g)=\tilde{\mathscr{E}}(f \cdot g)$ for all $i \leq a$ and $g \in[A]\left(\begin{array}{c}m \\ i\end{array}\right)$.

Observe that these notions are similar to those for ordinary graphs defined on sets. In particular, subgraphs are induced subgraphs. More precisely, if $(\tilde{m}, \tilde{\mathscr{E}})$ is an $\alpha$-graph and $f \in[A]\left(\begin{array}{c}\tilde{m} \\ m\end{array}\right)$ then $f$ induces the $\alpha$-graph $(m, \mathscr{E})$ by defining $\mathscr{E}(g)=\tilde{\mathscr{E}}(f \cdot g)$. Somehow it is not that apparent, and so let us stress this point, that we actually defined the analogue of ordered graphs. The reason is that parameter words yield a rigid representation of parameter sets.

Notation. For $\alpha$-graphs $(m, \mathscr{E})$ and $(\tilde{m}, \tilde{\mathscr{E}})$ we denote by $\left(\begin{array}{l}\tilde{\mathscr{E}} \\ \mathscr{E}\end{array}\right)$ the set of $\mathscr{E}$ subgraphs of $\tilde{\mathscr{E}}$, more precisely, $\left(\begin{array}{c}\tilde{\mathscr{E}} \\ \mathscr{E}\end{array}\right)=\left\{f \in[A]\left(\begin{array}{c}\tilde{m} \\ m\end{array}\right) \mid f: \mathscr{\mathscr { E }} \hookrightarrow \tilde{\mathscr{E}}\right\}$.

Apparently the notion of an induced partition theorem was first used by J. Spencer [1975] who proved an induced van der Waerden theorem. A more abstract, viz., categorical, framework for induced partition properties has been given in Deuber, Rothschild and Voigt [1982]. A complete Ramsey type partition theorem for $\alpha$-graphs over parameter sets has been proved first in Prömel [1985a]:

INDUCED GRAHAM-ROTHSCHILD THEOREM. Let $\mathscr{G}$ and $\mathscr{H}$ be $\alpha$-graphs and let $r$ be a positive integer. Then there exists an $\alpha$-graph $\mathscr{F}$ satisfying $\mathscr{F} \rightarrow(\mathscr{G})_{r}^{\mathscr{H}}$, meaning that for every coloring $\Delta:\left(\begin{array}{c}\mathscr{F} \\ \mathscr{P}\end{array}\right) \rightarrow r$ there exists an $f \in\left(\begin{array}{c}\mathscr{F} \\ \mathscr{G}\end{array}\right)$ such that $\Delta\rceil f \cdot\left(\begin{array}{l}\mathscr{G} \\ \mathscr{P}\end{array}\right)$ is a constant coloring, i.e., there exists a monochromatic $\mathscr{G}_{\text {-subgraph. }}$

This result is not that easy to prove, in fact, the original proof of Prömel [1985a] requires 20 pages in print. Recently, the particular case that at most one $\alpha_{i} \neq 0$ and, then, $\alpha_{i}=2$ and $\mathscr{H}:[A]\left(\begin{array}{l}k \\ i\end{array}\right) \rightarrow\{0,1\}$ being a constant mapping has been given a short proof in Frankl, Graham and Rödl [1987]. Using also some kind of amalgamation technique, Frankl, Graham and Rödl prove an induced and restricted result.

In $\S 12$ we show how our $*_{h}$-amalgamation can be used to give a relatively short proof of the induced Graham-Rothschild theorem.

11. Partite $\alpha$-graphs. In this section we adapt the notions introduced in $\S \S 5$ and 6. An $m$-partite $\alpha$-graph over $A^{m+n}$ is given by a mapping $\mathscr{E}:[A]\left(\begin{array}{c}m+n \\ i\end{array}\right) \rightarrow \alpha_{i}$ for $i \leq a$ such that $\mathscr{E}(f)>0$ implies that $f\rceil m \in[A]\left(\begin{array}{c}m \\ i\end{array}\right)$, i.e., every edge is crossing. Note that for every $i \leq a$ and $\xi<\alpha_{i}$ the $m$-partite $\alpha$-graph $\mathscr{E}$ defines an $m$-partite configuration $\mathscr{M}_{g \in[A]\left(\begin{array}{c}m \\ i\end{array}\right)}^{i, \xi} \subseteq A_{+}^{n}$ by

$$
\mathscr{M}_{g}^{i, \xi}=\left\{f \in A_{+}^{n} \mid \mathscr{E}(g \otimes f)=\xi\right\} .
$$

Moreover, for every $i \leq a$ and $g \in[A]\left(\begin{array}{c}m \\ i\end{array}\right)$ the set $A_{+}^{n}$ is split into mutually disjoint subsets $\left(\mathscr{M}_{g}^{i, \xi} \mid \xi<\alpha_{i}\right)$. So $\mathscr{E}$ is uniquely determined from the $m$-partite $i$-configurations $\mathscr{M}_{g \in[A]\left(\begin{array}{c}m \\ i\end{array}\right)}^{i, \xi}, i \leq a$ and $\xi<\alpha_{i}$. We refer to these configurations as the $m$-partite $i$-configurations associated with $\mathscr{E}$.

A partite embedding of an m-partite $\alpha$-graph $\mathscr{E}:[A]\left(\begin{array}{c}m+n \\ i\end{array}\right) \rightarrow \alpha_{i}$ into an $\tilde{m}$ partite $\alpha$-graph $\tilde{\mathscr{E}}:[A]\left(\begin{array}{c}\tilde{m}+\tilde{n} \\ i\end{array}\right) \rightarrow \alpha_{i}$ is given by a graph embedding $f \in[A]\left(\begin{array}{c}\tilde{m}+\tilde{n} \\ m+n\end{array}\right)$ such that $f\rceil \tilde{m} \in[A]\left(\begin{array}{c}\tilde{m} \\ m\end{array}\right)$. In other words, $f: \mathscr{M}_{g \in[A]\left(\begin{array}{c}m \\ i\end{array}\right)}^{i, \xi} \hookrightarrow \tilde{\mathscr{M}}_{g \in[A]\left(\begin{array}{c}\tilde{m} \\ i\end{array}\right)}^{i, \xi}$ for every $i \leq a$ and $\xi<\alpha_{i}$. 
By $\left(\begin{array}{l}\tilde{\mathscr{E}} \\ \mathscr{E}\end{array}\right)_{\text {part }}$ we denote the set of partite embeddings $f: \mathscr{E} \hookrightarrow \tilde{\mathscr{E}}$. This is well defined with respect to given $m$ and $\tilde{m}$. In the applications $m$ and $\tilde{m}$ will always be clear from the context.

Next we define the amalgamation of partite $\alpha$-graphs. Let $\mathscr{E}:[A]\left(\begin{array}{c}m+n \\ i\end{array}\right) \rightarrow \alpha_{i}$ for $i \leq a$ be an $m$-partite $\alpha$-graph. Let $h \in[A]\left(\begin{array}{c}m \\ k\end{array}\right)$. By $\mathscr{E}_{h}$ we denote the $k$-partite $\alpha$-graph on $A^{k+n}$ which is induced by $h$, i.e.,

$$
\mathscr{E}_{h}(g)=\mathscr{E}\left(\left(h \otimes\left(\lambda_{k}, \ldots, \lambda_{k+n-1}\right)\right) \cdot g\right)
$$

for every $g \in[A]\left(\begin{array}{c}k+n \\ i\end{array}\right), i \leq a$. Let $\mathscr{F}:[A]\left(\begin{array}{c}k+\tilde{n} \\ i\end{array}\right) \rightarrow \alpha_{i}$ be another $k$-partite $\alpha$ graph on $A^{k+\tilde{n}}$ and let $\mathbf{F}$ be a set of partite embeddings of $\mathscr{E}_{h}$ into $\mathscr{F}$. Say, $\mathbf{F}=\left\{e_{0}, \ldots, e_{z-1}\right\} \subseteq\left(\begin{array}{l}\mathscr{F} \\ \mathscr{E}_{h}\end{array}\right)_{\text {part }}$. We define $\mathscr{G}=\mathscr{E} *_{h}(\mathscr{F}, \mathbf{F})$, the amalgamation of $\mathscr{E}$ with $(\mathscr{F}, \mathbf{F})$ along $h$, which is an $m$-partite $\alpha$-graph on $A^{m+\tilde{n}+(z+1) \cdot m}$, by $\left.\mathscr{G}\left(e_{j}^{*} \cdot g \otimes f_{j} \cdot(g\rceil m\right)\right)=\mathscr{E}(g)$ for every $j<z, i \leq a$ and $g \in[A]\left(\begin{array}{c}m+n \\ i\end{array}\right)$, and $\mathscr{G}(g)=0$ in all other cases. Here $f_{j} \in[A]\left(\begin{array}{c}(z+1) \cdot m \\ m\end{array}\right)$ is as in Lemma 1 of $\S 6$ and $e_{j}^{*} \in[A]\left(\begin{array}{c}m+\tilde{n} \\ m+n\end{array}\right)$ is as supplied by Lemma 2 of $\S 6$. As $\mathscr{G}$ is nothing but a simultaneous amalgamation of all the $m$-partite $i$-configurations $\mathscr{M}_{g \in[A]\left(\begin{array}{c}m \\ i\end{array}\right)}^{i, \xi}$ one readily sees that the properties of the amalgamation of partite configurations listed in $\S 7$ carry over to the amalgamation of partite $\alpha$-graphs.

12. A proof of the induced Graham-Rothschild theorem. In this section we show how the method of $\S 8$ can be used to prove the induced Graham-Rothschild theorem (cf. $\S 10$ ). So let $\mathscr{G}$ and $\mathscr{H}$ be $\alpha$-graphs and let $r$ be a positive integer. We have to show the existence of an $\alpha$-graph $\mathscr{F}$ satisfying $\mathscr{F} \longrightarrow(\mathscr{G})_{r}^{\mathscr{H}}$. Say $\mathscr{H}$ is an $\alpha$-graph on $A^{k}$ and $\mathscr{G}$ is an $\alpha$-graph on $A^{m}$. Let $n$ be a positive integer satisfying $n \stackrel{[A]}{\rightarrow}(m)_{r}^{k}$. This exists by the Graham-Rothschild partition theorem for parameter sets. Let $\left(f_{\nu}\right)_{\nu<n_{0}^{0}}$ be an enumeration of $[A]\left(\begin{array}{l}n \\ m\end{array}\right)$. We define an $n$-partite $\alpha$-graph $\mathscr{E}^{0}$ on $A^{n+z \cdot(n+2)}$ by

$$
\mathscr{E}^{0}\left(f_{\nu} \cdot g \otimes x_{\nu}^{n_{0}^{0}} \cdot f_{\nu} \cdot g\right)=\mathscr{G}(g)
$$

for every $\nu<z, i \leq a$ and $g \in[A]\left(\begin{array}{c}m \\ i\end{array}\right)$, where $x_{\nu}^{n_{0}^{0}} \in[A]\left(\begin{array}{c}z \cdot(n+2) \\ n\end{array}\right)$ is defined in $\S 8$. Let $\mathscr{G}(g)=0$ in all other cases. Compare the analogous construction of $\mathscr{M}_{g \in[A]\left(\begin{array}{l}n \\ i\end{array}\right)}^{0}$ in $\S 8$. Now one applies an analogous procedure as in $\S 8$. For details compare $\S 8$.

We view $\mathscr{G}$ as a (crossing) $m$-partite $\alpha$-graph and $\mathscr{H}$ as a (crossing as well) $k$-partite $\alpha$-graph. Then

$$
\left(\begin{array}{c}
\mathscr{E}^{0} 0 \\
\mathscr{H}^{\circ}
\end{array}\right)_{\mathrm{part}} \subseteq\left\{f \otimes\left(x_{\nu}^{n_{0}^{0}} \cdot f\right) \mid \nu<n_{0}^{0}, f \in[A]\left(\begin{array}{l}
n \\
k
\end{array}\right)\right\}
$$

and

$$
\left(\begin{array}{c}
\mathscr{E}^{0} \\
\mathscr{G}
\end{array}\right)_{\text {part }}=\left\{f_{\nu} \otimes\left(x_{\nu}^{n_{0}^{0}} \cdot f_{\nu}\right) \mid \nu<n_{0}^{0}\right\}
$$

This is the analogue of Observation 3 from $\S 8$. Note that, by construction, also the analogue of Observation 4 is valid: if $\Delta:\left(\begin{array}{c}\mathscr{E}^{0} \\ \mathscr{H}\end{array}\right)_{\text {part }} \rightarrow r$ is such that $\Delta(g)=\Delta(\tilde{g})$ for all $g, \tilde{g} \in\left(\begin{array}{c}\mathscr{E}_{\mathscr{H}}^{0} \\ \mathbb{H}_{\text {part }}\end{array}\right.$ with $\left.\left.g\right\rceil n=\tilde{g}\right\rceil n$ then there exists a monochromatic $\mathscr{G}_{\text {-subgraph }}$ in $\left(\begin{array}{c}\mathscr{E}^{0} \\ \mathscr{G}\end{array}\right)_{\text {part }}$. 
Let $\left(h_{p}\right)_{p<q}$ be an enumeration of $[A]\left(\begin{array}{l}n \\ k\end{array}\right)$ and assume by induction that the $n$-partite $\alpha$-graph $\mathscr{E}^{p}$ has been constructed such that

(1) $\mathscr{M}_{g}^{i, \xi} \subseteq\left(\left\{x_{\nu}^{n^{p}} \mid \nu<n_{0}^{p}\right\} \otimes\left\{f_{\nu}^{h_{0}, n_{1}^{p}} \mid \nu<n_{1}^{p}\right\} \otimes \cdots \otimes\left\{f_{\nu}^{h_{p-1}, n_{p}^{p}} \mid \nu<n_{p}^{p}\right\}\right) \cdot g$ for every $i \leq a, g \in[A]\left(\begin{array}{l}n \\ i\end{array}\right)$ and $\xi<\alpha_{i}$ (where the $\mathscr{M}_{g \in[A]\left(\begin{array}{l}n \\ i\end{array}\right)}^{i, \xi}$ are the $n$-partite $i$-configurations associated with $\mathscr{E}^{p}$ ).

(2) For every coloring $\Delta:\left(\begin{array}{c}\mathscr{E}^{p} \\ \mathscr{C}\end{array}\right)_{\text {part }} \rightarrow r$ there exists an embedding $f \in\left(\begin{array}{l}\mathscr{E} p \\ \mathscr{E} 0\end{array}\right)_{\text {part }}$ such that $\Delta(f \cdot g)=\Delta(f \cdot \tilde{g})$ for all $g, \tilde{g} \in\left(\begin{array}{c}\mathscr{E}^{0} \\ \mathscr{K}\end{array}\right)_{\text {part }}$ with $\left.\left.g\right\rceil n=\tilde{g}\right\rceil n=h_{\mu}$ for some $\mu<p$. As in $\S 8$ we use the sparse product lemma to construct $\mathscr{E}^{p+1}$. So consider $h_{p} \in[A]\left(\begin{array}{l}n \\ k\end{array}\right)$ and define a $(p+1)$-partite graph $G$ on $\prod_{\mu \leq p} n_{\mu}^{p}$ by associating to each $\left(\nu_{0}, \ldots, \nu_{p}\right) \in \prod_{\mu \leq p} n_{\mu}^{p}$ the subgraph of $\mathscr{E}^{p}$ which is spanned by

$$
f_{h} \otimes\left(x_{\nu_{0}}^{n_{0}^{p}} \otimes f_{\nu_{1}}^{h_{0}, n_{1}^{p}} \otimes \cdots \otimes f_{\nu_{p}}^{h_{p-1}, n_{p}^{p}}\right) \cdot f_{h} .
$$

So $G$ is a $(p+1)$-partite graph on $\prod_{\mu \leq p} n_{\mu}^{p}$ with values in $\bigcup_{i \leq a}\left\{[A]\left(\begin{array}{l}k \\ i\end{array}\right) \rightarrow \alpha_{i}\right\}$.

By the sparse product lemma, this time with respect to $c=1$, there exists a $(p+1)$-partite graph $\tilde{G}$ on $\prod_{\mu \leq p} n_{\mu}^{p+1}$ with values in $\bigcup_{i \leq a}\left\{[A]\left(\begin{array}{c}k \\ i\end{array}\right) \rightarrow \alpha_{i}\right\}$ such that $I\left(\mathscr{H}^{\prime}\right)=\tilde{I}\left(\mathscr{H}^{\prime}\right)$ for all $\alpha$-graphs $\mathscr{H}^{\prime}$ on $A^{k}$, and such that for every $r$-coloring $\Delta: \tilde{G}^{-1}(\mathscr{H}) \rightarrow r$ there exists a monochromatic $G$-subgraph.

Let $n_{p+1}^{p+1}$ be the number of $G$-subgraphs of $\tilde{G}$, i.e., let $\prod_{\mu \leq p} X_{\mu}^{\nu} \in \prod_{\mu \leq p}\left[n_{\mu}^{p+1}\right]^{n_{\mu}^{p}}$, $\nu<n_{p+1}^{p+1}$ be an enumeration of all $G$-subgraphs of $\tilde{G}$. Now define a $k$-partite $\alpha$ graph $\mathscr{F}$ by

$$
\mathscr{F}\left(g \otimes\left(x_{\nu_{0}}^{n_{p+1}^{p+1}} \otimes f_{\nu_{1}}^{h_{0}, n_{1}^{p+1}} \otimes \cdots \otimes f_{\nu_{p}}^{h_{p-1}, n_{p}^{p+1}}\right) \cdot h_{p} \cdot g\right)=\tilde{G}\left(\nu_{0}, \ldots, \nu_{p}\right)(g)
$$

for every $\left(\nu_{0}, \ldots, \nu_{p}\right) \in \prod_{\mu \leq p} n_{\mu}^{p+1}$ and $g \in[A]\left(\begin{array}{l}k \\ i\end{array}\right), i \leq a$ and $\mathscr{F}(h)=0$ otherwise. This is a proper definition as $I\left(\mathscr{H}^{\prime}\right)=\tilde{I}\left(\mathscr{H}^{\prime}\right)$ for all $\alpha$-graphs $\mathscr{H}$ on $A^{k}$. For each $\nu<n_{p+1}^{p+1}$ define the embedding $e_{\nu}: \mathscr{E}_{h_{p}}^{p} \hookrightarrow \mathscr{F}$ by

$$
e_{\nu}=\left(\lambda_{0}, \ldots, \lambda_{k-1}\right) \tilde{\otimes}\left(x_{Y_{0}^{\nu}}^{n_{0}^{p+1}} \tilde{\otimes} f_{Y_{1}^{\nu}}^{h_{0}, n_{1}^{p+1}} \tilde{\otimes} \cdots \tilde{\otimes} f_{Y_{p}^{\nu}}^{h_{p-1}, n_{p}^{p+1}}\right)
$$

and let $\mathbf{E}=\left\{e_{\nu} \mid \nu<n_{p+1}^{p+1}\right\}$. Then let $\mathscr{E}^{p+1}=\mathscr{E}^{p} *_{h_{p}}(\mathscr{F}, \mathbf{E})$. As in $\S 8$ one checks that $\mathscr{E}^{p+1}$ again satisfies the inductive requirements. Eventually $\mathscr{E}^{q}$ has the desired properties, i.e. $\mathscr{E}^{q} \rightarrow(\mathscr{G})_{r}^{\mathscr{P}}$.

13. $\mathscr{H}$-connected $\alpha$-graphs. It turns out that the appropriate definition for the Sparse and Restricted Induced Graham-Rothschild Theorem is that of $\mathscr{H}$ connectedness.

Notation. Let $g \in[A]\left(\begin{array}{l}n \\ k\end{array}\right)$ and $h \in[A]\left(\begin{array}{c}n \\ k^{\prime}\end{array}\right)$ be two spaces in $A^{n}$. We say that $g$ and $h$ span the $m$-space $f \in[A]\left(\begin{array}{c}n \\ m\end{array}\right)$ if $\{g, h\} \subseteq f \cdot[A]\left(\begin{array}{c}m \\ k\end{array}\right) \cup f \cdot[A]\left(\begin{array}{c}m \\ k^{\prime}\end{array}\right)$ and for all $m^{\prime}<m$ and $f^{\prime} \in[A]\left(\begin{array}{c}n \\ m^{\prime}\end{array}\right)$ it follows that $\{g, h\} \nsubseteq f^{\prime} \cdot[A]\left(\begin{array}{c}m^{\prime} \\ k\end{array}\right) \cup f^{\prime} \cdot[A]\left(\begin{array}{c}m^{\prime} \\ k^{\prime}\end{array}\right)$ i.e., $f$ is minimal containing $g$ and $h$.

In this section let $i=0$ for $|A| \geq 2$ and $i=1$ for $|A|=1$. Recall that for $\alpha$-graphs $\mathscr{G}$ on $A^{m}$ the set $[A]\left(\begin{array}{c}m \\ i\end{array}\right)$ represents the set of vertices of $\mathscr{G}$. 
Notation. Let $\mathscr{G}$ be an $\alpha$-graph on $A^{m}$ and let $h$ and $h^{\prime}$ be vertices of $\mathscr{G}$, i.e., $h$, $h^{\prime} \in[A]\left(\begin{array}{c}m \\ i\end{array}\right)$. We say that $h$ and $h^{\prime}$ are neighbors if

(1) there exists $f \in[A]\left(\begin{array}{c}m \\ l\end{array}\right)$ for some $l \leq a$ such that $\mathscr{G}(f)>0$ and $\left\{h, h^{\prime}\right\} \subseteq$ $f \cdot[A]\left(\begin{array}{l}l \\ i\end{array}\right)$, or

(2) there exists $g \in[A]\left(\begin{array}{c}m \\ l\end{array}\right)$ for some $l \leq a$ such that $\mathscr{G}(g)>0$ and both $\{g, h\}$ and $\left\{g, h^{\prime}\right\}$ span the same subspace.

REMARK. Compared with ordinary graphs and hypergraphs which are defined on sets (1) is as expected, two vertices are adjacent if both belong to an existing edge (hyperedge, resp.). Condition (2) makes no sense for sets.

DEFINITION. Let $\mathscr{H}$ be an $\alpha$-graph on $A^{k}$ and let $\mathscr{G}$ be an $\alpha$-graph on $A^{m}$, such that $m \geq 2$ for $|A|=2$. We say that $\mathscr{G}$ is $\mathscr{H}$-connected if for all $f \in[A]\left(\begin{array}{c}m \\ k^{\prime}\end{array}\right)$ such that $\mathscr{G}_{f}$ is isomorphic to a weak subgraph of $H$ and such that any two distinct $h$, $h^{\prime} \in[A]\left(\begin{array}{c}m \\ i\end{array}\right) \backslash f \cdot[A]\left(\begin{array}{c}k^{\prime} \\ i\end{array}\right)$ can be joined by a path not using vertices from $f \cdot[A]\left(\begin{array}{c}k \\ i\end{array}\right)$, i.e., there exists a sequence $h=g_{0}, \ldots, g_{s}=h^{\prime} \in[A]\left(\begin{array}{c}m \\ i\end{array}\right) \backslash f \cdot[A]\left(\begin{array}{c}k \\ i\end{array}\right)$ such that $g_{t}$ and $g_{t+1}$ are neighbors for all $t<s$.

Here the notion of weak subgraphs is defined as follows: let $\mathscr{E}$, resp. $\tilde{\mathscr{E}}$, be $\alpha$-graphs on $A^{m}$, resp. $A^{\tilde{m}}$. An $f \in[A]\left(\begin{array}{c}\tilde{m} \\ m\end{array}\right)$ is a weak embedding of $\mathscr{E}$ into $\tilde{\mathscr{E}}$ if $\mathscr{E}(g) \in\{0, \mathscr{E}(f \cdot g)\}$ for all $g \in[A]\left(\begin{array}{c}m \\ l\end{array}\right), l \leq a$. In other words, some edges in $\mathscr{E}$ might be missing.

14. A sparse and restricted induced partition theorem for $\alpha$-graphs. This section contains the final result of our paper. For families $\mathbf{F}$ of $\alpha$-graphs we denote by $\operatorname{Forb}(\mathbf{F})$ the set of all $\alpha$-graphs not containing any member from $\mathbf{F}$ as a weak subgraph. Let $\mathscr{F}$ be an $\alpha$-graph on $A^{n}$, let $\mathscr{G}$ be an $\alpha$-graph on $A^{m}$ and let $\mathbf{G} \subseteq\left(\begin{array}{c}\mathscr{F} \\ \mathscr{G}\end{array}\right)$ be a family of $\mathscr{G}$-subgraphs of $\mathscr{F}$. In particular, $\mathbf{G} \subseteq[A]\left(\begin{array}{c}n \\ m\end{array}\right)$, so, forgetting the $\alpha$-structure, $\mathbf{G}$ can be viewed as a family of $m$-spaces in $A^{n}$. Thus the notion of $k$-girth $\mathbf{G}$, as defined in $\S 2$ makes sense.

SPARSE AND RESTRICTED INDUCED PARTITION THEOREM FOR $\alpha$-GRAPHS. Let $\mathscr{G}$ and $\mathscr{H}$ be $\alpha$-graphs and let $\mathbf{F}$ be a family of $\mathscr{H}$-connected $\alpha$-graphs such that $\mathscr{G}$ and $\mathscr{H}$ are members of $\mathrm{Forb}(\mathbf{F})$. Also let $r$ and $c$ be positive integers. Then there exists an $\alpha$-graph $\mathscr{F} \in \operatorname{Forb}(\mathbf{F})$ and there exists a family $\mathbf{G} \subseteq\left(\begin{array}{l}\mathscr{F} \\ \mathscr{G}\end{array}\right)$ of $\mathscr{G}$-subgraphs of $\mathscr{F}$ such that

(1) any two members of $\mathbf{G}$ intersect in an $\alpha$-graph which is isomorphic to a weak subgraph of $\mathscr{H}$, possibly the empty graph, moreover, say, $\mathscr{H}$ is an $\alpha$-graph on $A^{k}$., then any intersection of members of $\mathbf{G}$ which is a $k$-space induces an $\mathscr{H}$-graph (not just a weak subgraph);

$(2)(\mathscr{F}, \mathbf{G}) \rightarrow(\mathscr{G})_{r}^{\mathscr{H}}$, meaning that for every coloring $\Delta:\left(\begin{array}{l}\mathscr{F} \\ \mathscr{C}\end{array}\right) \rightarrow r$ there exists a $g \in \mathbf{G}$ such that $\Delta\rceil g \cdot\left(\begin{array}{c}\mathscr{G} \\ \mathscr{H}\end{array}\right)$ is a constant coloring;

(3) $k$-girth $\mathbf{G}>c$,

(4) if $\mathscr{G}$ itself is $\mathscr{H}$-connected then $\mathbf{G}=\left(\begin{array}{c}\mathscr{G} \\ \mathscr{G}\end{array}\right)$, i.e., in this case the family $\mathbf{G}$ can be chosen as the family of all $\mathscr{G}$-subgraphs of $\mathscr{F}$.

PROOF. With little changes we proceed essentially as described in $\S 12$. We point out the differences. The construction of $\mathscr{E}^{0}$ is somewhat different. Let $\mathscr{F}^{0}$ be an $\alpha$-graph such that $\mathscr{F}^{0} \rightarrow(\mathscr{G})_{r}^{\mathscr{H}}$. Such an $\mathscr{F}^{0}$ exists by the induced GrahamRothschild theorem. Say, $\mathscr{F}^{0}$ is an $\alpha$-graph on $A^{n}$. Let $\left(f_{\nu}\right)_{\nu<n_{0}^{0}}$ be an enumeration 
of $\left(\begin{array}{c}\mathscr{F}^{0} \\ \mathscr{G}\end{array}\right) \subseteq[A]\left(\begin{array}{c}n \\ m\end{array}\right)$ where, say, $\mathscr{G}$ is an $\alpha$-graph on $A^{m}$ by $\mathscr{E}^{0}\left(f_{\nu} \cdot g \otimes x_{\nu}^{n_{0}^{0}} \cdot f_{\nu} \cdot g\right)=\mathscr{G}(g)$ for every $\nu<n_{0}^{0}$ and $g \in[A]\left(\begin{array}{c}m \\ i\end{array}\right), i \leq a$, and $\mathscr{E}^{0}(g)=0$ otherwise. Observe that, alternatively, $\mathscr{E}^{0}$ could be defined as $\mathscr{E}^{0}\left(g \otimes x_{\nu}^{n_{0}^{0}} \cdot g\right)=\mathscr{F}^{0}(g)$ for all $g \in f_{\nu} \cdot[A]\left(\begin{array}{c}m \\ i\end{array}\right)$, so $\mathscr{E}^{0}$ obeys a kind of uniform description.

Let $\mathbf{G}^{0}=\left\{f_{\nu} \otimes x_{\nu}^{n_{0}^{0}} \cdot f_{\nu} \mid \nu<n_{0}^{0}\right\} \subseteq\left(\begin{array}{c}\mathscr{E}_{\mathscr{G}}^{0} \\ \mathscr{G}\end{array}\right)$. Let $\left(h_{p}\right)_{p<q}$ be an enumeration of $\left(\begin{array}{c}\mathscr{F}^{0} \\ \mathscr{H}^{\circ}\end{array}\right)$. Note that $\left(\begin{array}{c}\mathscr{F}^{0} 0 \\ \mathscr{C}^{\circ}\end{array}\right) \subseteq[A]\left(\begin{array}{l}n \\ k\end{array}\right)$, where, say, $\mathscr{H}$ is an $\alpha$-graph on $A^{k}$. Assume by induction that the $n$-partite $\alpha$-graph $\mathscr{E}^{p}$ and the family $\mathbf{G}^{p} \subseteq\left(\begin{array}{c}\mathscr{E}^{p} p \\ \mathscr{G}\end{array}\right)$ have been constructed such that

(1) $\mathscr{M}_{g}^{i, \xi} \subseteq\left(\left\{x_{\nu}^{n^{p}} \mid \nu<n_{0}^{p}\right\} \otimes\left\{f_{\nu}^{h_{0}, n_{1}^{p}} \mid \nu<n_{1}^{p}\right\} \otimes \cdots \otimes\left\{f_{\nu}^{h_{p-1}, h_{p}^{p}} \mid \nu<n_{p}^{p}\right\}\right) \cdot g$ for every $i \leq a, g \in[A]\left(\begin{array}{c}n \\ i\end{array}\right)$ and $\xi<\alpha_{i}$ (where the $\mathscr{M}_{g \in[A]\left(\begin{array}{c}n \\ i\end{array}\right)}^{i, \xi}$ are the $n$-partite $i$-configurations associated with $\mathscr{E}^{p}$ );

(2) for every coloring $\Delta:\left(\begin{array}{c}\mathscr{E}^{p} \\ \mathscr{C}\end{array}\right) \rightarrow r$ there exists an embedding $f \in\left(\begin{array}{l}\mathscr{E}^{p} \\ \mathscr{E} 0\end{array}\right)_{\text {part }}$ such that $f \cdot \mathbf{G}^{0} \subseteq \mathbf{G}^{p}$ and such that $\Delta(f \cdot g)=\Delta(f \cdot \tilde{g})$ for all $g, \tilde{g} \in\left(\begin{array}{l}\mathscr{C}_{\mathscr{C}}^{0} \\ )_{\text {part }}\end{array}\right.$ with $g\rceil n=\tilde{g}\rceil n=h_{\mu}$ for some $\mu<p$;

(3) $k$-girth $\mathbf{G}^{p}>c$;

(4) if $\mathscr{G}$ itself is $\mathscr{H}$-connected then $\mathbf{G}^{p}=\left(\begin{array}{c}\mathscr{E}^{p} \\ \mathscr{G}\end{array}\right)$;

(5) any two members of $\mathbf{G}^{p}$ intersect in an $\alpha$-graph which is isomorphic to a weak subgraph of $\mathscr{H}$, possibly the empty graph, however, any intersection in a $k$-space is an $\mathscr{H}$-graph;

(6) for every $i \leq a, g \in[A]\left(\begin{array}{c}n \\ i\end{array}\right)$ and $\xi \notin\left\{0, \mathscr{F}^{0}(g)\right\}$ we have that $\mathscr{M}_{g}^{i, \xi}=\varnothing$;

(7) $\mathscr{E}^{p} \in \operatorname{Forb}(\mathbf{F})$.

Clearly, $\left(\mathscr{E}^{0}, \mathbf{G}^{0}\right)$ satisfy (1)-(7).

We construct $\left(\mathscr{E}^{p+1}, \mathbf{G}^{p+1}\right)$ using the sparse product lemma. Consider $h_{p}$ and define the $(p+1)$-partite graph $G$ on $\prod_{\mu \leq p} n_{\mu}^{p}$ as in $\S 12$. Note that (6) implies that $G\left(\nu_{0}, \ldots, \nu_{p}\right)(g) \in\left\{0, \mathscr{F}^{0}\left(h_{p} \cdot g\right)\right\}$ for every $\left(\nu_{0}, \ldots, \nu_{p}\right) \in \prod_{\nu \leq p} n_{\nu}^{p}$ and $g \in[A]\left(\begin{array}{l}k \\ i\end{array}\right), i \leq a$. In other words, $G$ is a $(p+1)$-partite graph with values in $\bigcup_{i \leq a} \bigcup_{g \in[A]\left(\begin{array}{l}k \\ i\end{array}\right)}\left\{\{g\} \rightarrow\left\{0, \mathscr{F}^{0}\left(h_{p} \cdot g\right)\right\}\right\}$.

By the sparse product lemma there exists a $(p+1)$-partite graph $\tilde{G}$ on $\prod_{\mu \leq p} n_{\mu}^{p+1}$ with values in $\bigcup_{i \leq a} \bigcup_{g \in[A]\left(\begin{array}{c}k \\ i\end{array}\right)}\left\{\{g\} \rightarrow\left\{0, \mathscr{F}^{0}\left(h_{p} \cdot g\right)\right\}\right\}$ and there exists a family $\mathscr{S}$ of $G$-subgraphs with the usual properties. In particular, girth $\mathscr{S}>c$. As in $\S 12$, resp., $\S 8$, construct the $k$-partite $\alpha$-graph $\mathscr{F}$ together with the set $\mathbf{E}=\left\{e_{\nu} \mid \nu<n_{p+1}^{p+1}\right\}$ of embeddings $e_{\nu}: \mathscr{E}_{h_{p}}^{p} \hookrightarrow \mathscr{F}$. Then Observation 9 applies, in particular, $k$-girth $\mathbf{E}>c$. Also, any intersection of members of $\mathbf{E}$ in a $k$-space is an $\mathscr{H}$-graph.

The additional observations about $\mathscr{F}$ are (say $\mathscr{F}$ is a $k$-partite $\alpha$-graph on $\left.A^{k+\tilde{m}}\right)$ :

OBSERVATION 10. $\mathscr{F}_{h}$ can be weakly embedded into $\mathscr{H}$ for every $h \in[A]\left(\begin{array}{c}k+\tilde{m} \\ k\end{array}\right)$ such that $h\rceil k \in[A]\left(\begin{array}{l}k \\ k\end{array}\right)$.

ProOF. Recall that $h_{p}: \mathscr{H} \mathscr{F}^{0}$ and $\tilde{G}$ is a $(p+1)$-partite graph with values in $\bigcup_{i \leq a} \bigcup_{g \in[A]\left(\begin{array}{l}k \\ i\end{array}\right)}\left\{\{g\} \rightarrow\left\{0, \mathscr{F}^{0}\left(h_{p} \cdot g\right)\right\}\right\}$. 
OBSERVATION 11. For every $h \in[A]\left(\begin{array}{c}k+\tilde{m} \\ j\end{array}\right)$ such that $\left.h\right\rceil k \notin[A]\left(\begin{array}{l}k \\ j\end{array}\right)$ and $j>1$ if $|A|=1$ (i.e., $\mathscr{F}_{h}$ is not just a vertex) the subgraph $\mathscr{F}_{h}$ is not connected, in particular, it is not $\mathscr{H}$-connected.

PROOF. Consider any vertex $h \cdot \tilde{g}$ of $\mathscr{F}_{h}$ such that $\left.h \cdot \tilde{g}\right\rceil k \in[A]\left(\begin{array}{l}k \\ 0\end{array}\right)$. As $\mathscr{F}(g)=0$ for all noncrossing $g$ we conclude that $h \cdot \tilde{g}$ is not a neighbor of any vertex of $\mathscr{F}_{h}$, i.e., $h \cdot \tilde{g}$ is isolated.

COROLlary. $\mathscr{F}_{h} \in \operatorname{Forb}(\mathbf{F})$.

Now we define

$$
\mathscr{E}^{p+1}=\mathscr{E}^{p} *_{h_{p}}(\mathscr{F}, \mathbf{E}) \text { and } \mathbf{G}^{p+1}=\mathbf{G}^{p} *_{h_{p}} \mathbf{E} .
$$

We check (1)-(7).

(5) is satisfied by Observation 10 ,

(3) follows from the girth property of *-amalgamation,

(2) follows from the coloring property of $*$-amalgamation and Observation 7 ,

(4) and (7) follow from the next observation (compare the restrictedness property of *-amalgamation)

OBSERVATION 12. Assume that every two members of $\mathbf{E}$ intersect in an $\alpha$ graph which is a weak subgraph of $\mathscr{H}$ (which is satisfied by construction); then any $\mathscr{H}$-connected subgraph of $\mathscr{E}^{p+1}$ already is contained in one of the $\mathscr{E}^{p, \nu}$ for $\nu<n_{p+1}^{p+1}$, where $\mathscr{E}^{p, \nu}=\mathscr{E}_{\vec{\lambda} \otimes e_{\nu}^{*} \otimes f_{\nu}}^{p+1}$.

PROOF. By the amalgamation property any two distinct $\mathscr{E} p, \nu$ and $\mathscr{E}^{p, \nu^{\prime}}$ intersect in a crossing subgraph of $\mathscr{F}$. By Lemma 1 vertices $\mathscr{E}^{p, \nu}$ and $\mathscr{E}^{p, \nu^{\prime}}$ both not belonging to the intersection can be joined only via the intersection, contradicting $\mathscr{H}$-connectedness.

(1) follows (as in §12) immediately from the definition of the amalgamation

(6) follows from the construction of $\mathscr{F}$.

Finally, then, $\mathscr{E}^{q}$ and $\mathbf{G}^{q}$ have the desired properties.

REMARKS. (1) An analogous result for ordinary graphs and hypergraphs defined on sets has been established in Prömel and Voigt [1987]. In this paper also an example is given which shows that some kind of connectedness has to be required to guarantee assertion (4).

(2) This result extends the Frankl-Graham-Rödl [1987] result.

15. Other structures. Cum grano salis, our methods apply also to other structures such as affine, resp. projective, spaces over finite fields. The interested reader can easily figure out details. Compare, e.g., Graham, Leeb and Rothschild [1972], Spencer [1979], Deuber, Voigt [1982], Prömel [1986], Frankl, Graham and Rödl [1987].

\section{REFERENCES}

[1973] A. Brauer, Gedenkrede auf Issai Schur, Issai Schur, Gesammelte Abhandlungen (A. Brauer and H. Rohrbach, eds.), Springer, Berlin, 1973, V-XIV.

[1984] W. Deuber, H. J. Prömel, B. L. Rothschild and B. Voigt, A restricted version of HalesJewett's theorem, Finite and Infinite Sets (A. Hajnal, L. Lovász and V. T. Sós, eds.), Colloq. Math. Soc. János Bolyai 37, North-Holland, Amsterdam, 1984, pp. 231-246.

[1982] W. Deuber, B. L. Rothschild and B. Voigt, Induced partition theorems, J. Combin. Theory Ser. A 32 (1982), 225-240. 
[1982] W. Deuber and B. Voigt, Partitionseigenschaften endlicher affiner und projektiver Räume, European J. Combin. 3 (1982), 329-340.

[1983] _ Der Satz von van der Waerden über arithmetische Progressionen, Jber. Deutsch. Math.Verein. 85 (1983), 66-85.

[1987] P. Frankl, R. L. Graham and V. Rödl, Induced restricted Ramsey theorems for spaces, J. Combin. Theory Ser. A 44 (1987), 120-128.

[1971] _ Ramsey's theorem for n-parameter sets, Trans. Amer. Math. Soc. 159 (1971), 257-292.

[1980] R. L. Graham, B. L. Rothschild and J. H. Spencer, Ramsey theory, Wiley, New York, 1980.

[1963] A. W. Hales and R. I. Jewett, Regularity and positional games, Trans. Amer. Math. Soc. 106 (1963), 222-229.

[1976] J. Nesetł̌il and V. Rödl, Van der Waerden's theorem for sequences of integers not containing an arithmetic progression of $k$ terms, Comment. Math. Univ. Carolinae 17 (1976), 675-688.

[1983] _ Another proof of the Folkman-Rado-Sanders theorem, J. Combin. Theory Ser. A 34 (1983), 108-109.

[1984] _ Sparse Ramsey graphs, Combinatorica 4 (1984), 71-78.

[1986a] _ On sets of integers with the Schur property, Graphs and Combinatorics 2 (1986), 269-275.

[1986b] _ Finite union theorem with restrictions, Graphs and Combinatorics 2 (1986), 357-361.

[1985] H. J. Prömel, Induced partition properties of combinatorial cubes, J. Combin. Theory Ser. A 39 (1985), 177-208.

[1986a] _ _ Partition properties of q-hypergraphs, J. Combin. Theory Ser. B 41 (1986), 356-385.

[1986] H. J. Prömel and B. Voigt, Graham Rothschild parameter sets, The Mathematics of Ramsey Theory (J. Nesetřll and V. Rödl, eds.), Springer-Verlag, Berlin, Heildberg and New York (to appear).

[1987] _ Ramsey theorems for finite graphs. I, Report No 86447-OR, Institut für Operations Research, Univ. Bonn, 1987.

[1933] R. Rado, Studien zur Kombinatorik, Math. Z. 36 (1933), 424-480.

[1981] V. Rödl, On Ramsey families of sets, unpublished manuscript.

[1986] _ Personal communication.

[xx] A. Rucinski and B. Voigt, in preparation.

[1917] I. Schur, Über die Kongruenz $x^{m}+y^{m}=z^{m}(\bmod p)$, Jber. Deutsch. Math.-Verein. 25 (1917), 114-117.

[1975] J. Spencer, Restricted Ramsey configurations, J. Combin. Theory Ser. A 19 (1975), 278-286.

[1979] _ Ramsey's theorem for spaces, Trans. Amer. Math. Soc. 249 (1979), 363-371.

[1927] B. L. van der Waerden, Beweis einer Baudetschen Vermutung, Nieuw Arch. Wisk. 15 (1927), 212-216.

INSTITUT FÜR OPERATIONS RESEARCH, UNIVERSITÄT BONN, NASSESTRASSE 2, 5300 BONN, FRG

Fakultat für Mathematik, Universität Bielefeld, 4800 Bielefeld, FRG 